\title{
A compendium of monocyte transcriptome datasets to foster
}

\section{biomedical knowledge discovery [version 1; peer review: 1}

\section{approved, 1 approved with reservations]}

\section{Darawan Rinchai1, Sabri Boughorbel (i)2, Scott Presnell33, Charlie Quinn3, Damien Chaussabel (iD)}

\footnotetext{
${ }^{1}$ Systems Biology Department, Sidra Medical and Research Center, Doha, Qatar ${ }^{2}$ Biomedical informatics, Sidra Medical and Research Center, Doha, Qatar

${ }^{3}$ Benaroya Research Institute at Virginia Mason, Seattle, USA
}

\author{
V1 First published: 07 Mar 2016, 5:291 \\ https://doi.org/10.12688/f1000research.8182.1 \\ Latest published: 25 Apr 2016, 5:291 \\ https://doi.org/10.12688/f1000research.8182.2
}

Open Peer Review

Approval Status

1

2

version 2

(revision)

25 Apr 2016

version 1

07 Mar 2016

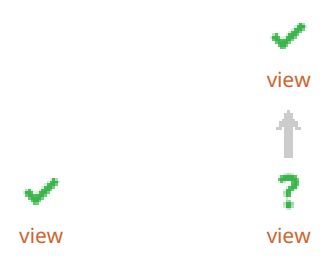

1. Marc Pellegrini, Walter and Eliza Hall

Institute of Medical Research, Parkville,

Australia

2. Ping Chen, National Institutes of Health,

Besthesda, USA

David Kuo, National Institutes of Health, Bethesda, USA

University of California San Diego, La Jolla,

USA

Any reports and responses or comments on the article can be found at the end of the article.

This article is included in the Sidra Medicine

(ال) Sidra Medicine gateway. 
This article is included in the Data: Use and

Reuse collection.

Corresponding author: Darawan Rinchai (drinchai@sidra.org)

Competing interests: No competing interests were disclosed.

Grant information: DR, SB and DC received support from the Qatar Foundation.

The funders had no role in study design, data collection and analysis, decision to publish, or preparation of the manuscript.

Copyright: (c) 2016 Rinchai $D$ et al. This is an open access article distributed under the terms of the Creative Commons Attribution License, which permits unrestricted use, distribution, and reproduction in any medium, provided the original work is properly cited.

How to cite this article: Rinchai D, Boughorbel S, Presnell S et al. A compendium of monocyte transcriptome datasets to foster biomedical knowledge discovery [version 1; peer review: 1 approved, 1 approved with reservations] F1000Research 2016, 5:291 https://doi.org/10.12688/f1000research.8182.1

First published: 07 Mar 2016, 5:291 https://doi.org/10.12688/f1000research.8182.1 


\section{Introduction}

Platforms such as microarrays and, more recently, next generation sequencing have been leveraged to generate molecular profiles at the scale of entire systems. The global perspective gained using such approaches is potentially transformative. Transcriptome profiling enabled for instance the characterization of molecular perturbations that occur in the context of a wide range disease processes $^{1-10}$. This in turn has provided opportunities for the discovery of biomarkers and for the development of novel therapeutic modalities ${ }^{3,11-13}$. More recently such systems-scale profiling of the blood transcriptome has also been used to monitor response to vaccines or therapeutic drugs ${ }^{14-19}$. The democratization of these approaches has led to proliferation of data in public repositories: over 1.7 million individual transcriptome profiles from more than 65,000 studies have been deposited to date in the NCBI Gene Expression Omnibus (GEO), a public repository of transcriptome profiles.

Taken together this vast body of "collective data" holds the promise of accelerating the pace of biomedical discovery by creating countless opportunities for identifying and filling critical knowledge gaps. Building tools that provide biomedical researchers with the ability to seamlessly interact with collections of datasets along with rich contextual information is essential in promoting insight and enabling knowledge discovery. To address this need we have developed an interactive data browsing and visualization web application, the Gene Expression Browser (GXB).

GXB was described in a recent publication and is available as open source software on GitHub ${ }^{20}$. This tool constitutes a simple interface for the browsing and interactive visualization of large volumes of heterogeneous data. Users can easily customize data plots by adding multiple layers of information, modifying the order of samples, and generating links that capture these settings which can be inserted in email communications or in publications. Accessing the tool via these links also provides access to rich contextual information that is essential for data interpretation. This includes access to gene information and relevant literature, study design information, detailed sample information as well as ancillary data ${ }^{20}$.

In recent years, a large number of transcriptional studies have been conducted aiming at the characterization and functional classification of monocytes in health and disease. Monocytes are a population of immune cells found in the blood, bone marrow, and spleen. They constitute $\sim 10 \%$ of the total circulating blood leukocytes in humans. They can remain in the blood circulation for up to 1-2 days, after which time, if they have not been recruited to a tissue, they die and are removed. They are considered the systemic reservoir of myeloid precursors for renewal of tissue macrophages and dendritic cells. Monocytes play a key role during immune response as professional phagocytes ${ }^{21,22}$, and producers of immune mediators ${ }^{23,24}$. Indeed, reports show that monocytes are recruited at the site of infections as innate effectors of the inflammatory response to microbes, killing pathogens via phagocytosis, production of reactive oxygen intermediate $(\mathrm{ROIs})^{25}$, reactive nitrogen intermediate $(\mathrm{RNIs})^{26,27}$, myeloperoxidase (MPO) ${ }^{28,29}$, and producing inflammatory cytokines ${ }^{30}$ that contribute to further amplifying the antimicrobial response ${ }^{31}$.
Human monocytes are derived from hematopoietic stem cells in the bone marrow and are released into peripheral blood circulation upon maturation. They are divided into three major subsets based on the expression of cell surface markers CD14 and CD16. The most prevalent subset in the blood circulation, accounting for $90 \%$ of all monocytes, are the classical monocytes that express high levels of CD14 but low levels of CD16. The remaining 10\% is divided into two subsets: intermediate monocyte with high expression of CD14 and CD16 (CD14+CD16+) and non-classical monocytes that express low levels of CD14 but high levels of CD16 (CD14dimCD16++ or CD14+CD16++ $)^{32-34}$.

In this data note we are making available via GXB a curated compendium of 93 public datasets relevant to human monocyte immunobiology, representing a total of 4,516 transcriptome profiles.

\section{Materials and methods}

\section{Identification of monocyte datasets}

Potentially relevant datasets deposited in GEO were identified using an advanced query based on the Bioconductor package GEOmetadb and the SQLite database that captures detailed information on the GEO data structure; https://www.bioconductor.org/packages/release/bioc/html/GEOmetadb.html ${ }^{35}$. The search query was designed to retrieve entries where the title and description contained the word Monocyte OR Monocytes, were generated from human samples, using Illumina or Affymetrix commercial platforms. The query result is appended with rich metadata from GEOmetadb that allows for manual filtering of the retrieved collection.

The relevance of each entry returned by this query was assessed individually. This process involved reading through the descriptions and examining the list of available samples and their annotations. Sometimes it was also necessary to review the original published report in which the design of the study and generation of the dataset is described in more detail. The datasets cover a broad range of human immunology studies investigating monocyte immunobiology in the context of diseases and through comparison with diverse cell populations and study types as illustrated by a graphical representation of relative occurrences of terms in the list of diseases loaded into our tool (Figure 1). A wide range of cell types and diseases are represented. Ultimately, the collection was comprised of 93 curated datasets. It includes datasets generated from studies profiling primary human CD14+ cells isolated from patients with autoimmune diseases (7), bacterial, virus and parasite infections (7), cancer (4), cardiovascular diseases (4), kidney diseases (4), as well as monocytes isolated from healthy subjects (58) (Figure 2). The 58 datasets in which monocytes were isolated from healthy subjects were classified based on whether profiling was conducted ex vivo or following in vitro experiments. In total 38 datasets were identified in which primary human CD14+ cells were stimulated or infected in in vitro experiments (Figure 2). Among the many noteworthy datasets, there are 8 datasets investigating differences between monocytes subsets; classical (CD14++CD16-), intermediate $(\mathrm{CD} 14+\mathrm{CD} 16+)$ and non-classical monocytes (CD14-CD16++) ${ }^{32-34}$ [GXB: GSE16836, GSE18565, GSE25913, GSE34515, GSE35457, GSE51997, GSE60601, GSE66936]. Another dataset from Banchereau and colleagues investigated responses of monocyte and dendritic cells to 13 different vaccines 


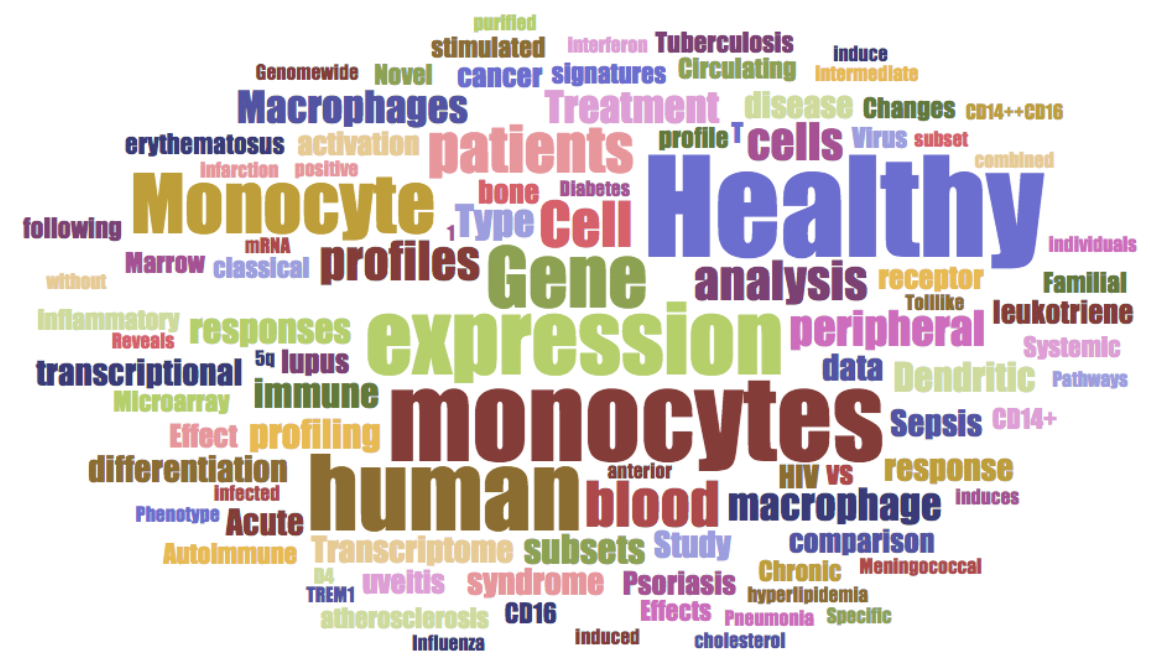

Figure 1. Thematic composition of the dataset collection. Word frequencies extracted from text descriptions of the studies loaded into the GXB tool are depicted as a word cloud. The size of the words is proportional to their frequency.

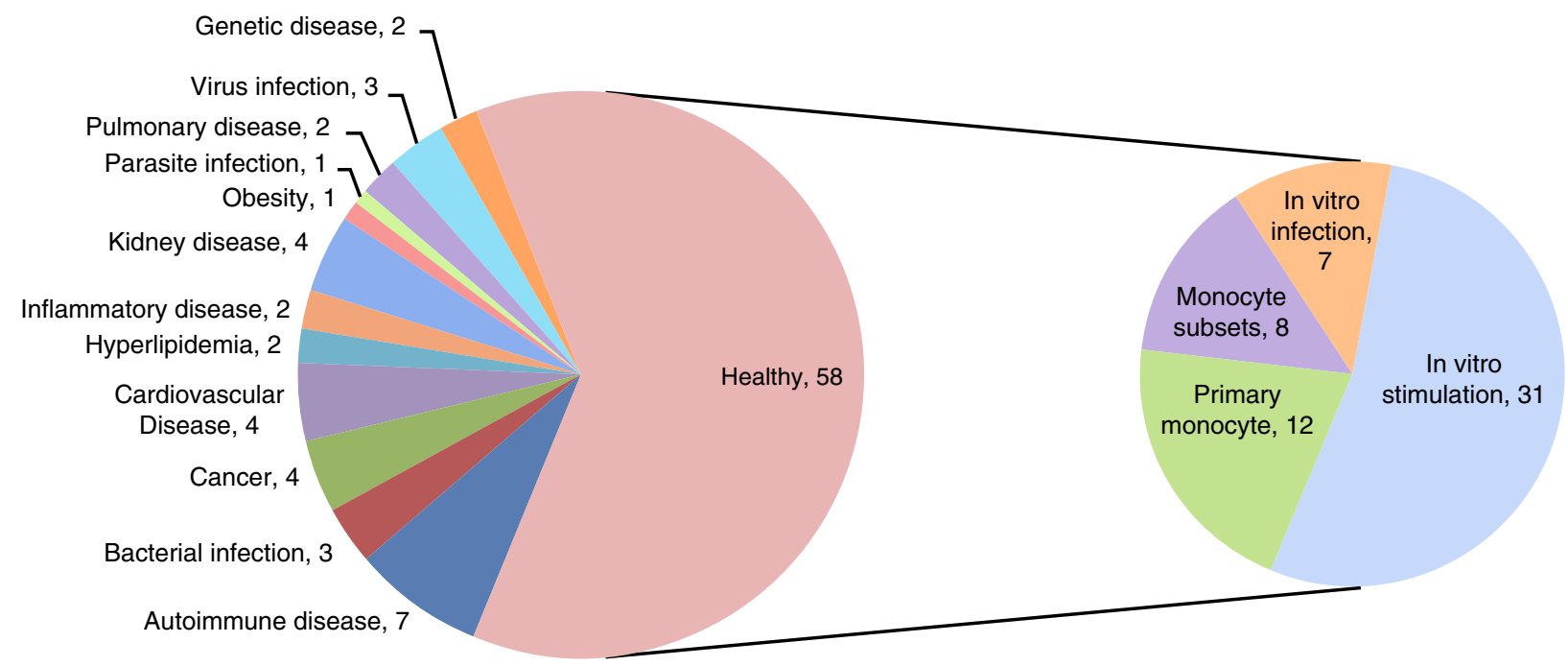

Figure 2. Break down of the dataset collection by category. The pie chart on the left panel indicates dataset frequencies by disease status. The chart on the right panel indicates the type of studies carried out for the 58 datasets consisting of monocyte obtained exclusively from healthy donors.

in vitro ${ }^{36}$ [GXB: GSE44721]. The datasets that comprise our collection are listed in Table 1 and can be browsed interactively in GXB.

\section{Dataset upload and annotation on GXB}

Once a final selection had been made each dataset was downloaded from GEO in the SOFT file format. It was in turn uploaded on an instance of the Gene Expression Browser (GXB) hosted on the Amazon Web Services cloud. Available sample and study information were also uploaded. Samples were grouped according to possible interpretations of study results and ranking based on the different group comparisons that were computed (e.g. comparing monocyte isolated from case vs controls in studies where profiling was performed ex-vivo; or stimulated vs medium control in in vitro experiments).

\section{Short Gene Expression Brower tutorial}

The GXB software has been described in detail in a recent publication $^{20}$. This custom software interface provides users with a means to easily navigate and filter the dataset collection available at http://monocyte.gxbsidra.org/dm3/landing.gsp. A web tutorial is also available online: http://monocyte.gxbsidra.org/dm3/tutorials.gsp\#gxbtut. Briefly, datasets of interest can be quickly identified either by filtering using criteria from pre-defined lists on the left or by entering a query term in the search box at the top of the dataset navigation page. Clicking on one of the studies listed in the dataset navigation page opens a viewer designed to provide interactive browsing and graphic representations of large-scale data in an interpretable format. This interface is designed to present ranked gene lists and display expression results graphically in a 


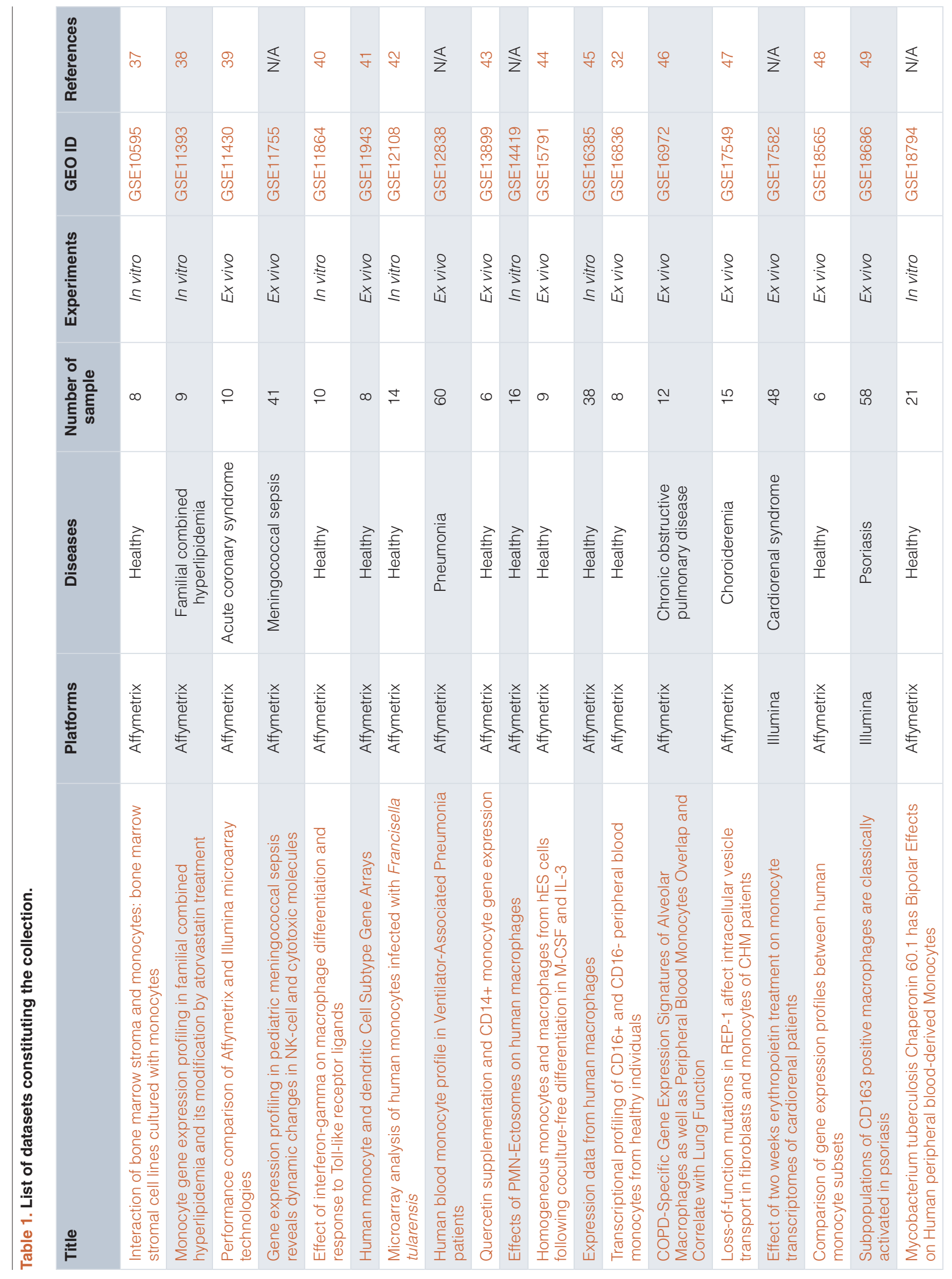




\begin{tabular}{|c|c|c|c|c|c|c|c|c|c|c|c|c|c|c|c|c|c|c|c|}
\hline لֶّ & $\mp$ & 요 & $\overline{5}$ & న్ & $\frac{b}{z}$ & م & है & ले & $\stackrel{\$}{z}$ & 员 & 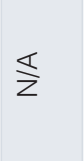 & i̊n & 占 & 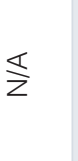 & in & $\stackrel{\infty}{n}$ & هి & 8 & $\frac{1}{z}$ \\
\hline $\begin{array}{l}\text { 으 } \\
\text { 없 }\end{array}$ & 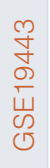 & 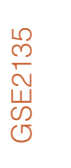 & 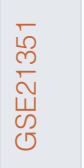 & 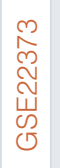 & 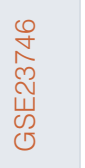 & 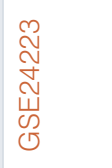 & $\begin{array}{l}\text { ᄋ } \\
\infty \\
\stackrel{\infty}{4} \\
\underset{0}{0}\end{array}$ & 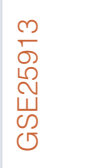 & 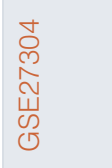 & 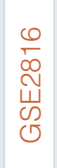 & 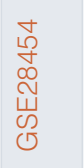 & & 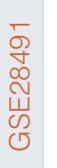 & 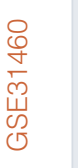 & 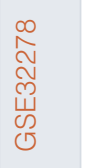 & 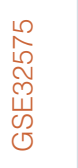 & 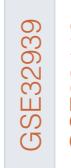 & 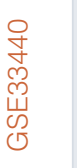 & 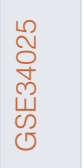 \\
\hline 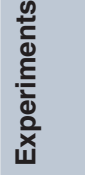 & $\begin{array}{l}\stackrel{0}{3} \\
\stackrel{x}{x}\end{array}$ & 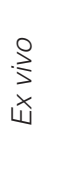 & $\begin{array}{l}\stackrel{0}{2} \\
\stackrel{x}{凶}\end{array}$ & 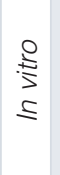 & $\begin{array}{l}\stackrel{0}{3} \\
\underset{4}{4}\end{array}$ & 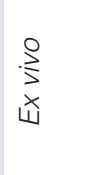 & $\begin{array}{l}\frac{2}{5} \\
\frac{5}{5} \\
=\end{array}$ & $\begin{array}{l}\stackrel{0}{3} \\
\underset{x}{凶}\end{array}$ & $\begin{array}{l}\stackrel{\rho}{3} \\
\underset{x}{4}\end{array}$ & 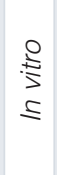 & $\begin{array}{l}\stackrel{0}{3} \\
\text { 出 }\end{array}$ & & $\begin{array}{l}\stackrel{P}{>} \\
\text { 㐫 }\end{array}$ & 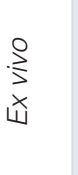 & 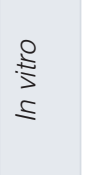 & $\begin{array}{l}\stackrel{0}{3} \\
\text { 崫 }\end{array}$ & 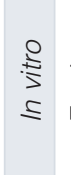 & $\begin{array}{l}\stackrel{?}{>} \\
\text { 崩 }\end{array}$ & $\begin{array}{l}\text { o } \\
\text { s. } \\
\text { s }\end{array}$ \\
\hline 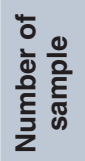 & 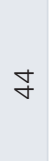 & $\stackrel{\nabla}{\square}$ & 0 & 0 & ஜூ & $\stackrel{\Omega}{\stackrel{\Omega}{L}}$ & 으 & $\stackrel{+}{\sim}$ & $\stackrel{\llcorner}{\leftarrow}$ & 0 & $\stackrel{\varrho}{R}$ & fे & $\stackrel{m}{m}$ & 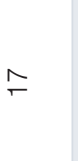 & $\infty$ & $\stackrel{\infty}{q}$ & 0 & $\approx$ & $\stackrel{\varphi}{\circ}$ \\
\hline 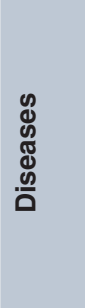 & $\begin{array}{l}\frac{\frac{0}{0}}{0} \\
\frac{0}{3} \\
\frac{0}{0} \\
\frac{0}{5} \\
\frac{0}{5}\end{array}$ & 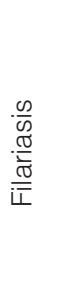 & $\begin{array}{l}\frac{\vec{I}}{\mathbb{T}} \\
\frac{\mathbb{\Phi}}{I}\end{array}$ & $\begin{array}{l}\frac{\vec{I}}{\mathbb{E}} \\
\frac{\mathbb{d}}{I} \\
\frac{\mathbb{O}}{1}\end{array}$ & 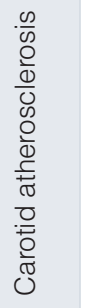 & 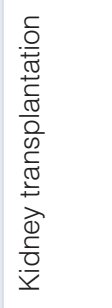 & $\begin{array}{l}\frac{\widehat{I}}{\mathbb{I}} \\
\frac{\mathbb{O}}{I}\end{array}$ & 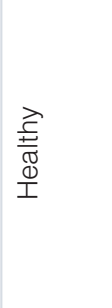 & 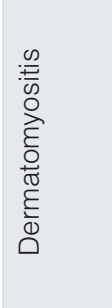 & $\begin{array}{l}\overrightarrow{\vec{I}} \\
\frac{\underline{I}}{\mathbb{\pi}} \\
\stackrel{\mathbb{T}}{I}\end{array}$ & 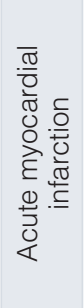 & 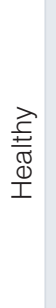 & 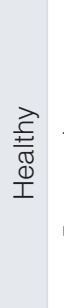 & 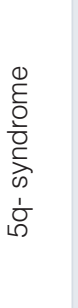 & $\begin{array}{l}\vec{I} \\
\frac{ \pm}{\mathbb{N}} \\
\frac{\mathbb{N}}{I}\end{array}$ & $\begin{array}{l}\frac{3}{0} \\
\mathbb{0} \\
\frac{0}{0} \\
0\end{array}$ & 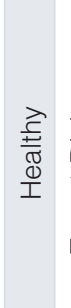 & 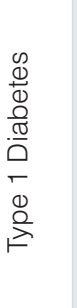 & 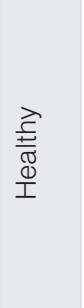 \\
\hline $\begin{array}{l}\frac{0}{5} \\
\frac{5}{0} \\
\frac{\text { के }}{\alpha}\end{array}$ & 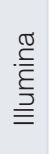 & 离 & 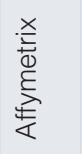 & 宏 & 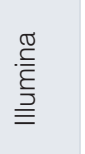 & 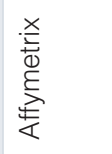 & 肴 & 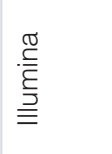 & 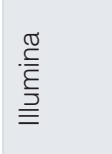 & 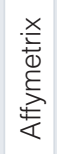 & $\begin{array}{l}\stackrel{\sqrt[\sigma]{\xi}}{\underline{\underline{\xi}}} \\
\underline{\underline{\underline{\underline{y}}}}\end{array}$ & & 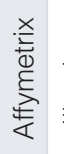 & 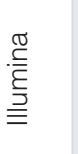 & 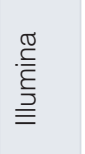 & 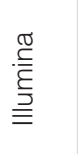 & 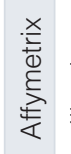 & 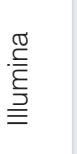 & 竞 \\
\hline 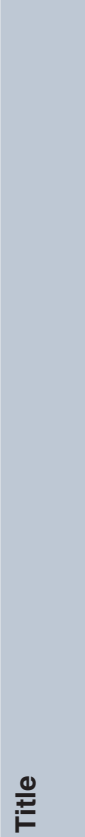 & 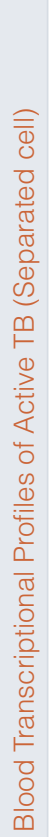 & 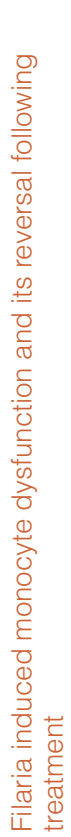 & 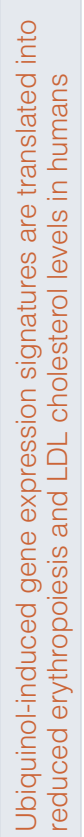 & 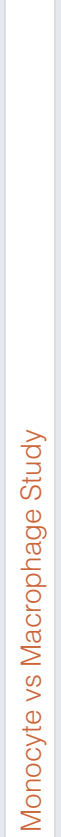 & 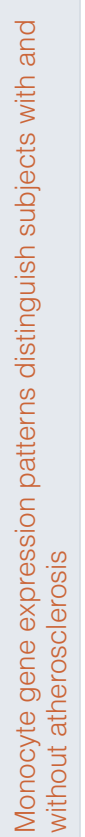 & 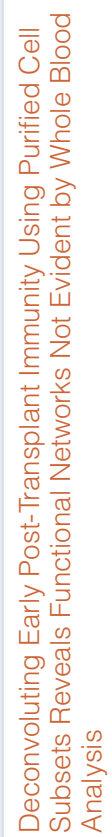 & 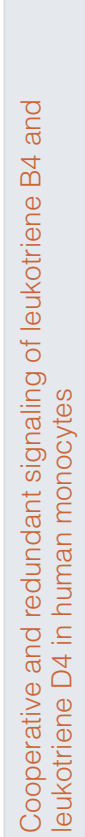 & 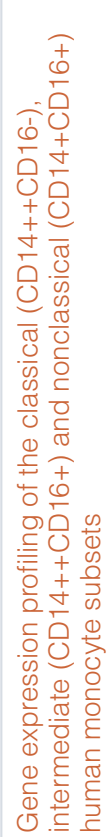 & 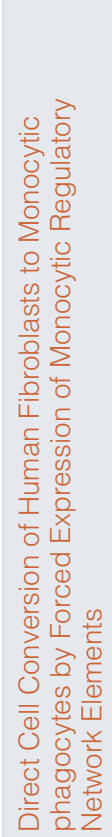 & 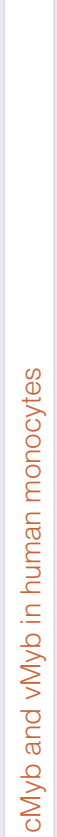 & 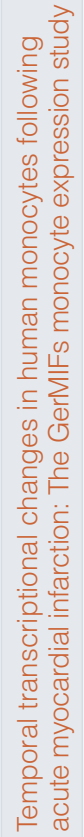 & 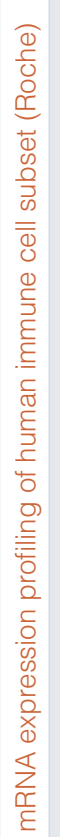 & 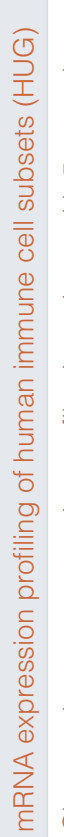 & 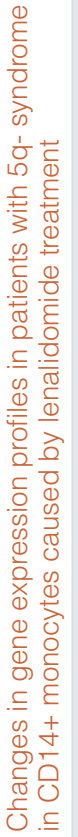 & 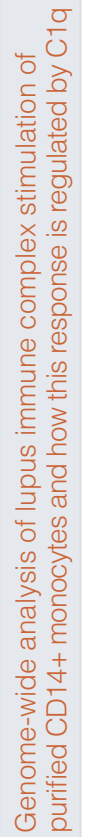 & 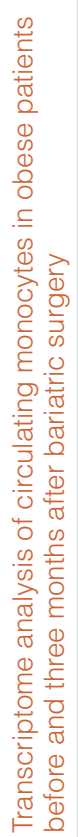 & 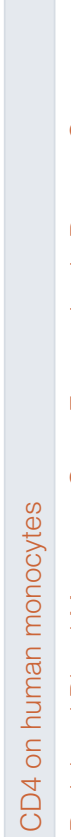 & 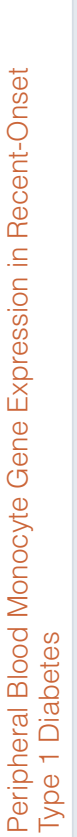 & 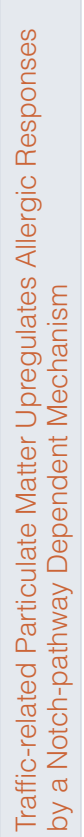 \\
\hline
\end{tabular}




\begin{tabular}{|c|c|c|c|c|c|c|c|c|c|c|c|c|c|c|c|c|c|c|c|c|}
\hline ֻٕ & $\overline{0}$ & $\widetilde{\varphi}$ & $\frac{\mathbb{z}}{z}$ & రె & $\frac{1}{z}$ & ठै & $\stackrel{8}{0}$ & 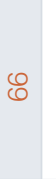 & $\frac{\pi}{z}$ & $\frac{1}{z}$ & $\hat{\theta}$ & $\frac{1}{z}$ & 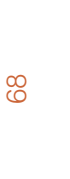 & 8 & $\frac{\$}{z}$ & ㅇ & @ & $\bar{\kappa}$ & $\cong$ & $\stackrel{m}{\wedge}$ \\
\hline 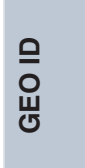 & 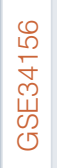 & 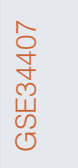 & 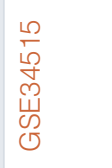 & 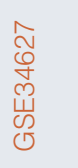 & $\begin{array}{l}\infty \\
\stackrel{0}{0} \\
心 \\
心 \\
心 \\
心\end{array}$ & 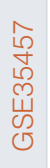 & 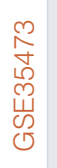 & 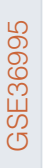 & 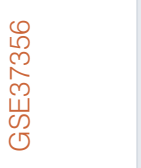 & 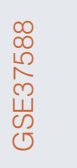 & 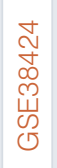 & 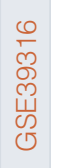 & 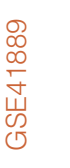 & 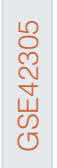 & 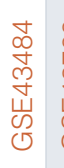 & 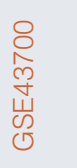 & 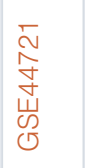 & 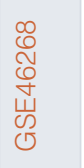 & 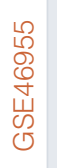 & 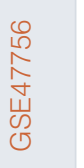 \\
\hline 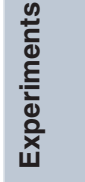 & $\begin{array}{l}\text { 量 } \\
\text { s } \\
\text { I }\end{array}$ & $\begin{array}{l}\frac{9}{5} \\
\frac{5}{2} \\
\underline{5}\end{array}$ & $\begin{array}{l}\stackrel{9}{3} \\
\underset{4}{4}\end{array}$ & $\begin{array}{l}\frac{0}{\frac{2}{3}} \\
\leq \\
\leq\end{array}$ & $\begin{array}{l}\stackrel{9}{5} \\
\stackrel{5}{5}\end{array}$ & $\begin{array}{l}\stackrel{0}{3} \\
\stackrel{x}{x}\end{array}$ & $\begin{array}{l}\stackrel{0}{5} \\
\stackrel{5}{5} \\
\leq\end{array}$ & $\begin{array}{l}\stackrel{0}{\stackrel{3}{3}} \\
\text { 出 }\end{array}$ & $\begin{array}{l}\stackrel{0}{3} \\
\text { 出 }\end{array}$ & $\begin{array}{l}\stackrel{0}{\frac{2}{3}} \\
\leq \\
\text { s. }\end{array}$ & 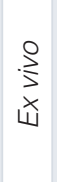 & $\begin{array}{l}\stackrel{9}{\frac{9}{3}} \\
\leq\end{array}$ & 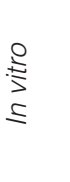 & $\begin{array}{l}\frac{9}{2} \\
\frac{5}{3} \\
=\end{array}$ & 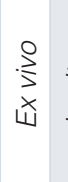 & $\begin{array}{l}\stackrel{0}{\frac{0}{3}} \\
\leq\end{array}$ & $\begin{array}{l}\stackrel{?}{\frac{9}{3}} \\
\text { s }\end{array}$ & 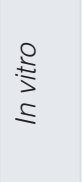 & 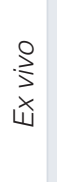 & $\begin{array}{l}\stackrel{0}{2} \\
\stackrel{x}{\mid x}\end{array}$ \\
\hline 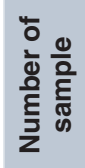 & 过 & $\infty$ & $\sigma$ & $\stackrel{\simeq}{\simeq}$ & $\stackrel{\simeq}{\simeq}$ & g & 0 & 0 & $\stackrel{\sim}{\curvearrowright}$ & 0 & $\infty$ & ৪ & $\stackrel{\Xi}{\sim}$ & $\stackrel{\llcorner}{\sim}$ & 0 & $\infty$ & 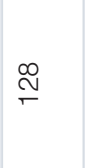 & $\stackrel{\simeq}{\leftarrow}$ & $\underset{\forall}{\forall}$ & 'ె \\
\hline 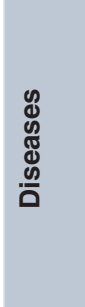 & $\begin{array}{l}\stackrel{\vec{I}}{\frac{I}{\pi}} \\
\stackrel{\mathbb{\Phi}}{I}\end{array}$ & $\begin{array}{l}\overrightarrow{\vec{I}} \\
\stackrel{\underline{\Xi}}{\widetilde{J}} \\
\stackrel{\Phi}{I}\end{array}$ & 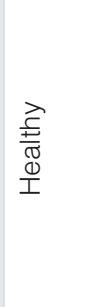 & 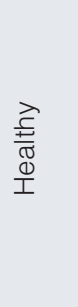 & 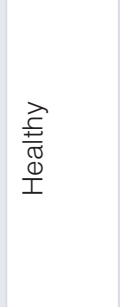 & $\begin{array}{l}\overrightarrow{\frac{1}{ \pm}} \\
\frac{\mathbb{N}}{\Phi} \\
\frac{\mathbb{1}}{I}\end{array}$ & $\begin{array}{l}\stackrel{\vec{I}}{\underline{I}} \\
\frac{\mathbb{d}}{I} \\
\stackrel{0}{I}\end{array}$ & 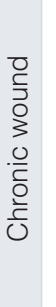 & 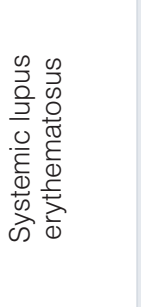 & 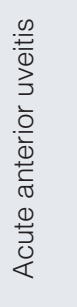 & 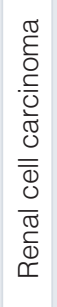 & $\begin{array}{l}\text { 蒿 } \\
\frac{\mathbb{J}}{I} \\
\frac{\mathbb{I}}{I}\end{array}$ & $\begin{array}{l}\text { 章 } \\
\frac{\mathbb{d}}{I} \\
\frac{\mathbb{D}}{I}\end{array}$ & $\begin{array}{l}\frac{\infty}{0} \\
\frac{\pi}{0} \\
\frac{0}{0} \\
0 \\
0\end{array}$ & 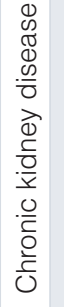 & 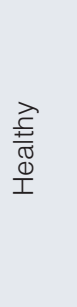 & 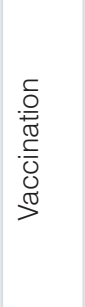 & $\begin{array}{l}\frac{\vec{I}}{\mathbb{E}} \\
\stackrel{\mathbb{D}}{I}\end{array}$ & $\begin{array}{l}\frac{\infty}{\infty} \\
\frac{D}{0} \\
\stackrel{\infty}{\infty}\end{array}$ & $\begin{array}{l}\overline{0} \\
0 \\
\overline{0} \\
0 \\
\bar{\sigma} \\
\frac{\pi}{0} \\
\frac{0}{0} \\
\frac{0}{0} \\
0\end{array}$ \\
\hline 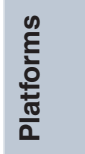 & 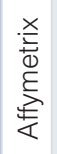 & 竞 & 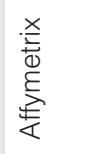 & 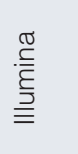 & 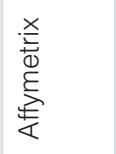 & $\begin{array}{l}\stackrel{\sqrt[\varpi]{\xi}}{\underline{\underline{\xi}}} \\
\underline{\underline{\underline{y}}}\end{array}$ & 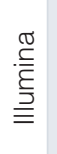 & 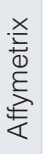 & $\begin{array}{l}\stackrel{\sqrt[\sigma]{\frac{\sigma}{\xi}}}{\underline{\underline{\underline{\rho}}}} \\
\end{array}$ & 肴 & 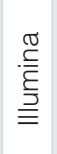 & 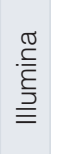 & 﨎 & 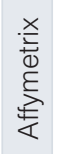 & 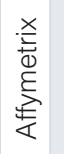 & 竞 & $\begin{array}{l}\stackrel{\sqrt[\sigma]{\underline{E}}}{\underline{\underline{\xi}}} \\
\underline{\underline{\underline{\underline{y}}}}\end{array}$ & 离 & 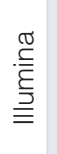 & 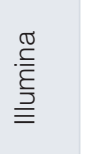 \\
\hline (0) & 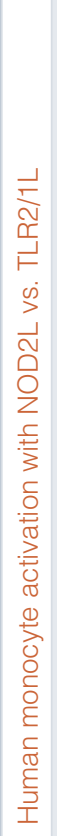 & 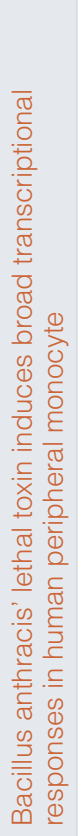 & 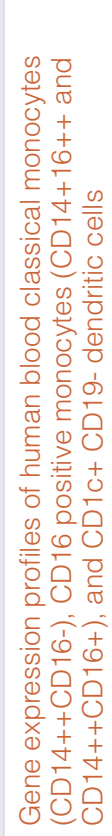 & 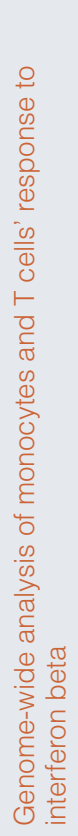 & 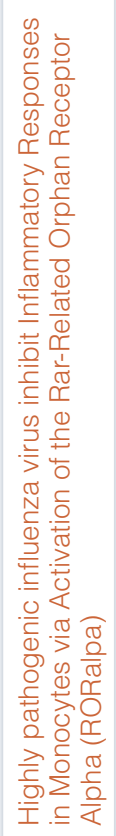 & 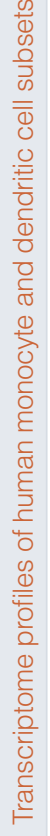 & 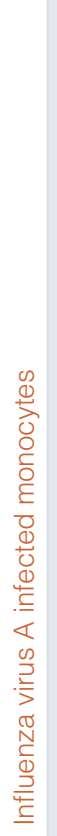 & 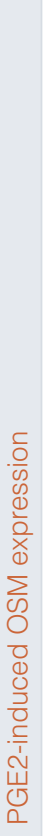 & 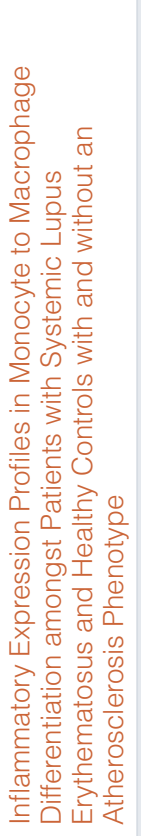 & 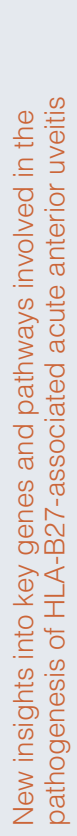 & 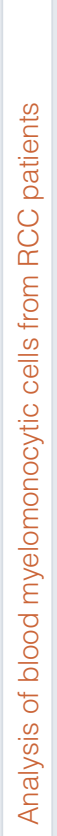 & 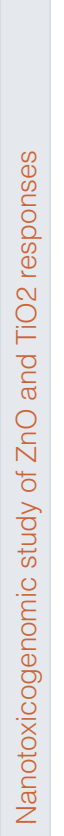 & 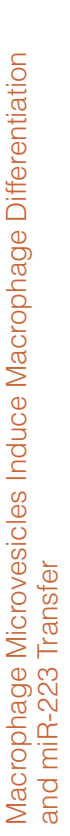 & 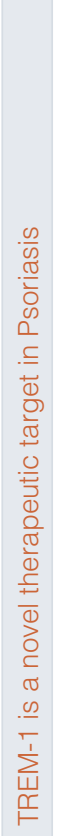 & 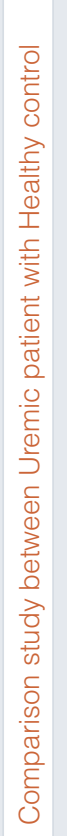 & 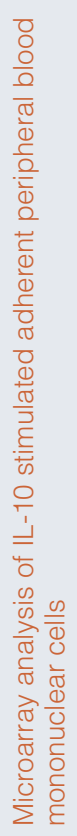 & 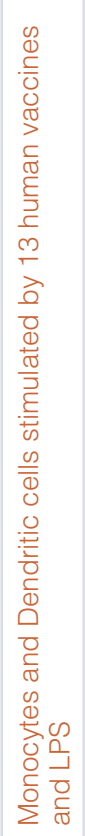 & 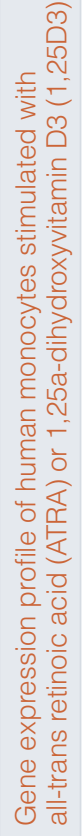 & 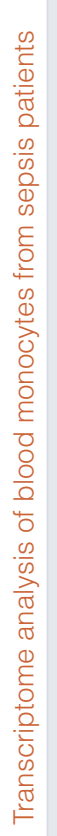 & 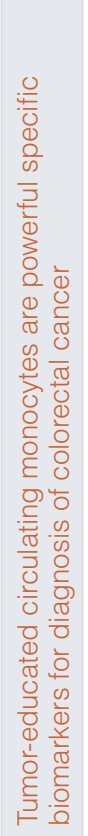 \\
\hline
\end{tabular}




\begin{tabular}{|c|c|c|c|c|c|c|c|c|c|c|c|c|c|c|c|c|c|c|c|}
\hline 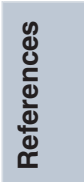 & $\frac{N}{N}$ & $\frac{\mathbb{s}}{z}$ & $\stackrel{L}{\wedge}$ & $\frac{\mathbb{s}}{z}$ & $\stackrel{\bullet}{\wedge}$ & $\hat{\wedge}$ & $\stackrel{\infty}{\wedge}$ & $\frac{\mathbb{L}}{z}$ & $\stackrel{2}{1}$ & 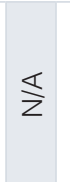 & $\infty$ & $\bar{\infty}$ & & $\stackrel{\sim}{\sim}$ & $\stackrel{\nwarrow}{z}$ & $\infty$ & $\frac{\mathbb{s}}{z}$ & $\stackrel{\S}{z}$ & 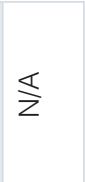 \\
\hline $\begin{array}{l}\text { 응 } \\
\text { 엉 }\end{array}$ & 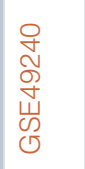 & 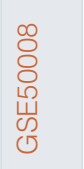 & $\begin{array}{l}\bar{\tau} \\
\overline{8} \\
\stackrel{\leftrightarrow}{4} \\
心 \\
心\end{array}$ & 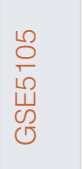 & 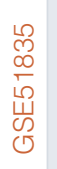 & 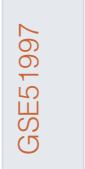 & 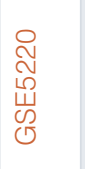 & 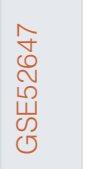 & 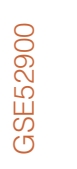 & 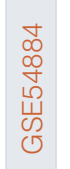 & 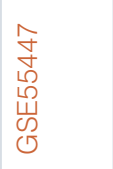 & 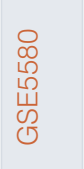 & 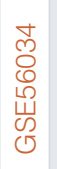 & 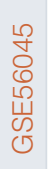 & 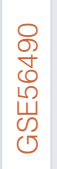 & 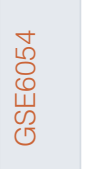 & $\begin{array}{l}\overline{8} \\
8 \\
8 \\
\mathbb{1} \\
0 \\
0\end{array}$ & 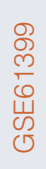 & 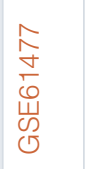 \\
\hline 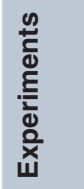 & $\begin{array}{l}\stackrel{9}{5} \\
\text { s. } \\
\leq\end{array}$ & $\begin{array}{l}\stackrel{0}{3} \\
\text { 出 }\end{array}$ & $\begin{array}{l}\stackrel{0}{3} \\
\text { 出 }\end{array}$ & $\begin{array}{l}\stackrel{0}{3} \\
\text { 出 }\end{array}$ & 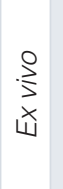 & $\begin{array}{l}\stackrel{\rho}{3} \\
\text { 出 }\end{array}$ & $\begin{array}{l}\stackrel{0}{3} \\
\stackrel{x}{x}\end{array}$ & 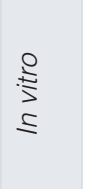 & $\begin{array}{l}\stackrel{0}{>} \\
\text { 出 }\end{array}$ & $\begin{array}{l}\frac{9}{3} \\
\leq \\
\leq\end{array}$ & $\begin{array}{l}\stackrel{0}{3} \\
\stackrel{x}{x}\end{array}$ & $\begin{array}{l}\stackrel{\rho}{3} \\
\text { 出 }\end{array}$ & $\begin{array}{l}\stackrel{?}{3} \\
\text { 出 }\end{array}$ & $\begin{array}{l}\stackrel{0}{3} \\
\text { 嵌 }\end{array}$ & $\begin{array}{l}\frac{5}{3} \\
\frac{5}{5} \\
\frac{9}{2}\end{array}$ & $\begin{array}{l}\stackrel{0}{\stackrel{3}{3}} \\
\text { 爻 }\end{array}$ & 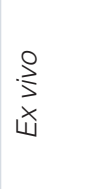 & $\begin{array}{l}\stackrel{\rho}{3} \\
\text { 出 }\end{array}$ & 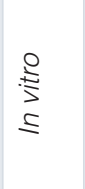 \\
\hline 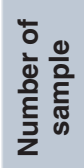 & $\stackrel{\simeq}{\simeq}$ & \& & $\infty$ & $\mathscr{8}$ & 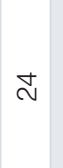 & @) & $\stackrel{\varphi}{\sim}$ & $\stackrel{\simeq}{\sim}$ & $\stackrel{\nabla}{\ulcorner}$ & 0 & $\stackrel{\infty}{\sim}$ & $\stackrel{\Im}{\Im}$ & $\stackrel{\llcorner}{\stackrel{\infty}{q}}$ & ิ & $\stackrel{\varphi}{\leftarrow}$ & $\stackrel{\sim}{N}$ & $\simeq$ & iి & $\cong$ \\
\hline $\begin{array}{l}\mathscr{Q} \\
\Phi \\
\Phi \\
\Phi \\
\Phi \\
口\end{array}$ & $\begin{array}{l}\vec{T} \\
\frac{\vec{I}}{\mathbb{W}} \\
\frac{\mathbb{T}}{I}\end{array}$ & 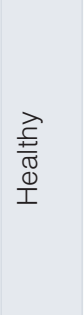 & 主 & 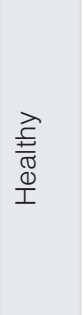 & 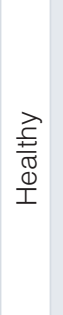 & 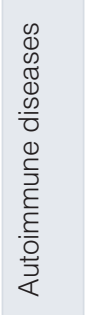 & 主 & 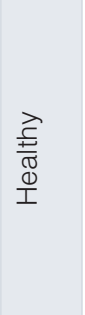 & $\gtreqless$ & $\begin{array}{l}\frac{\vec{I}}{\frac{I}{\pi}} \\
\frac{\mathbb{I}}{I}\end{array}$ & 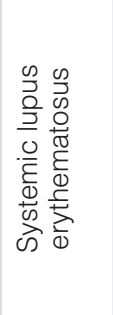 & 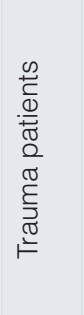 & & 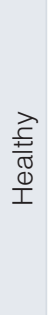 & 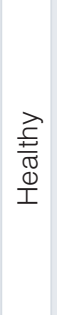 & 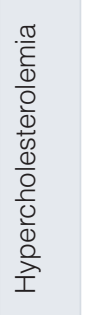 & 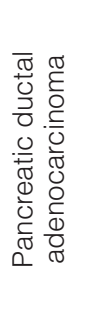 & 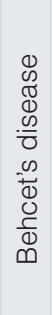 & 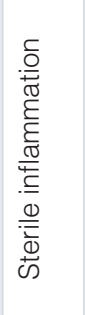 \\
\hline 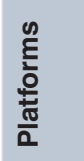 & 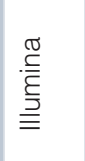 & 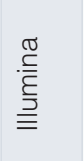 & 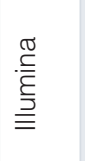 & 﨎 & 离 & 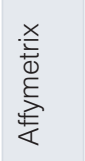 & 景 & 竝 & 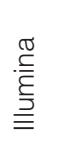 & 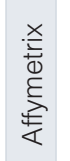 & 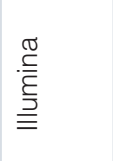 & 齐 & 景 & 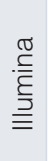 & 景 & 景 & 竞 & 颃 & $\begin{array}{l}\stackrel{\sqrt[\sigma]{\xi}}{\underline{\underline{\xi}}} \\
\underline{\underline{\underline{y}}}\end{array}$ \\
\hline$\stackrel{\oplus}{=}$ & 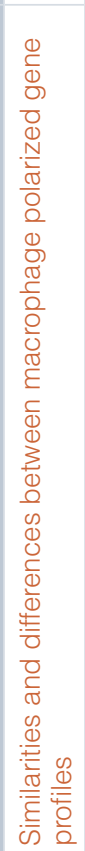 & 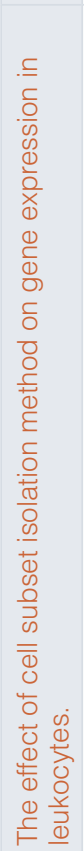 & 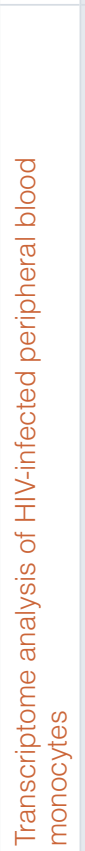 & 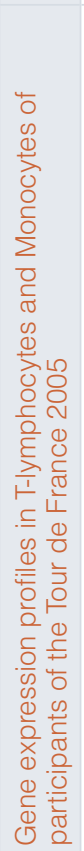 & 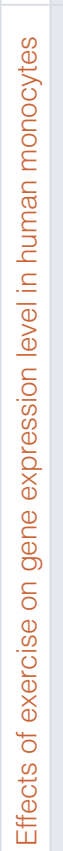 & 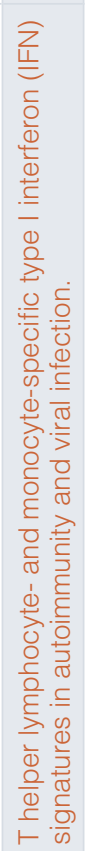 & 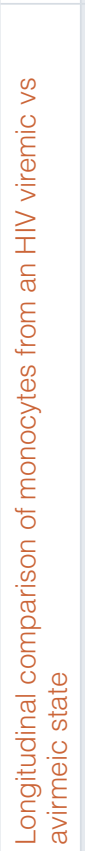 & 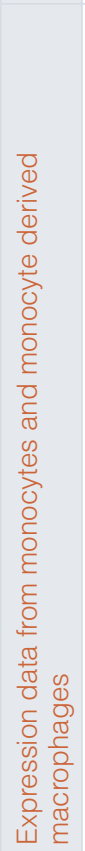 & 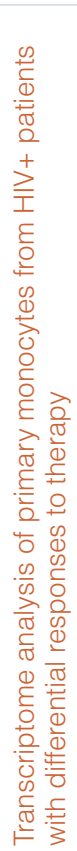 & 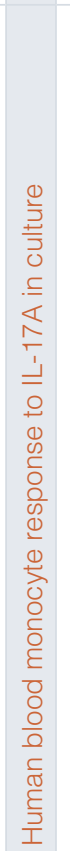 & 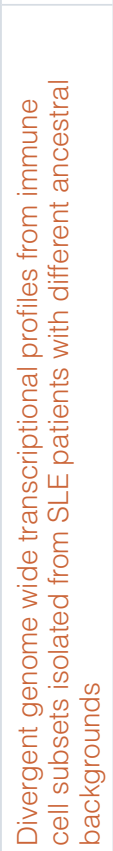 & 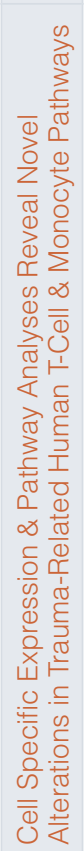 & 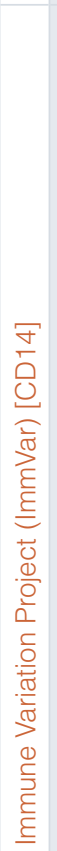 & 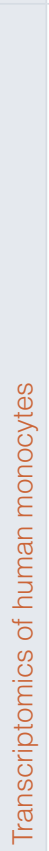 & 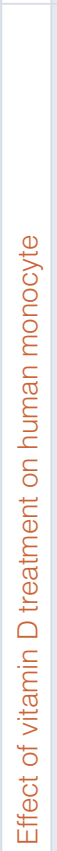 & 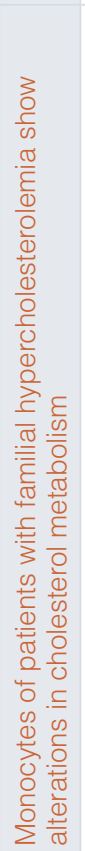 & 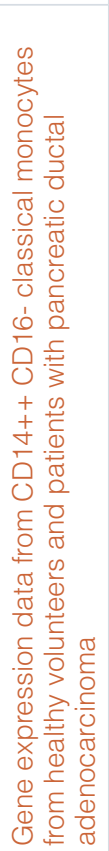 & 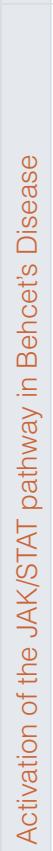 & 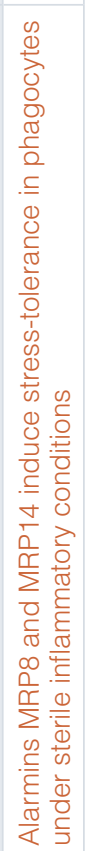 \\
\hline
\end{tabular}




\begin{tabular}{|c|c|c|c|c|c|c|c|c|c|c|c|c|c|c|c|c|}
\hline 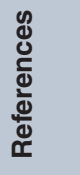 & \ం & $\stackrel{\llcorner}{\infty}$ & 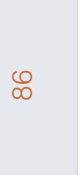 & $\underset{\Sigma}{\Sigma}$ & $\hat{\infty}$ & $\begin{array}{l}\infty \\
\infty\end{array}$ & வ & $\underset{z}{\Sigma}$ & ৪ి & б & ๙ૅ & $\stackrel{\mathbb{Z}}{\Sigma}$ & ஜ & $\frac{1}{z}$ & ठా & $\stackrel{\leftrightarrow}{\circ}$ \\
\hline $\begin{array}{l}\text { 응 } \\
\text { 원 }\end{array}$ & 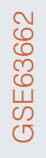 & 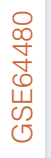 & 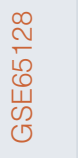 & 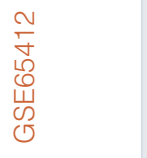 & 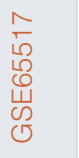 & 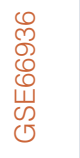 & $\begin{array}{l}\infty \\
\stackrel{\infty}{T} \\
\stackrel{\omega}{\omega} \\
\widetilde{N}\end{array}$ & 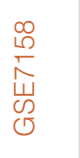 & 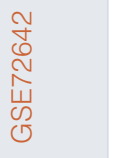 & 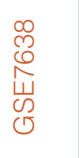 & $\begin{array}{l}\hat{0} \\
\infty \\
\tilde{\omega} \\
心 \\
0\end{array}$ & $\begin{array}{l}0 \\
\stackrel{\infty}{ } \\
\sim \\
\infty \\
山 \\
心 ్\end{array}$ & 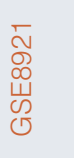 & 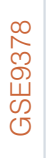 & 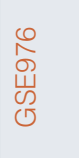 & 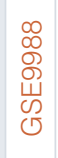 \\
\hline 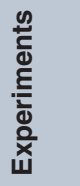 & 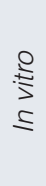 & $\begin{array}{l}\stackrel{0}{\frac{5}{3}} \\
\text { s }\end{array}$ & $\begin{array}{l}\stackrel{0}{3} \\
\text { 爻 }\end{array}$ & 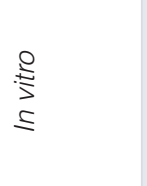 & $\begin{array}{l}\stackrel{0}{>} \\
\text { 出 }\end{array}$ & $\begin{array}{l}\stackrel{\rho}{2} \\
\stackrel{x}{凶}\end{array}$ & 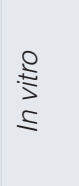 & $\begin{array}{l}\stackrel{\rho}{3} \\
\stackrel{x}{凶}\end{array}$ & $\begin{array}{l}\stackrel{0}{3} \\
\text { 幽 }\end{array}$ & $\begin{array}{l}\stackrel{0}{\stackrel{3}{2}} \\
\text { 爻 }\end{array}$ & $\begin{array}{l}\text { ? } \\
\text { : } \\
\text { s } \\
\text { s. }\end{array}$ & 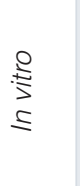 & 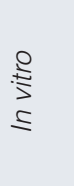 & 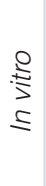 & 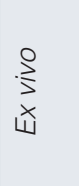 & 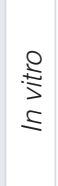 \\
\hline 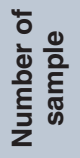 & 0 & $\sigma$ & ల్ల & ฉ & $\stackrel{m}{\square}$ & $\bar{\sim}$ & $\approx$ & $\stackrel{\sim}{\sim}$ & $\stackrel{\infty}{-}$ & 8 & $\infty$ & $\sigma$ & 유 & 으 & $\stackrel{d}{\sim}$ & $\mp$ \\
\hline $\begin{array}{l}\mathscr{J} \\
\mathscr{D} \\
\mathbb{J} \\
.0 \\
0\end{array}$ & 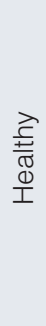 & 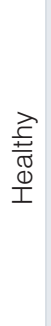 & $\begin{array}{l}\stackrel{\vec{I}}{\underline{\underline{T}}} \\
\stackrel{\mathbb{I}}{I}\end{array}$ & 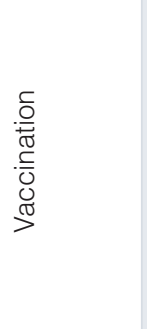 & 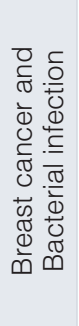 & 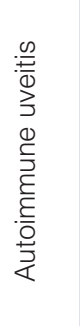 & 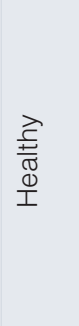 & 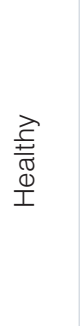 & 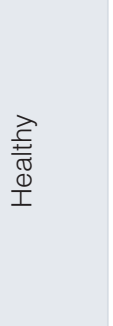 & 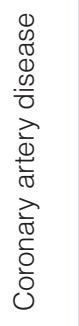 & $\begin{array}{l}\stackrel{\vec{E}}{\underline{\mathbb{E}}} \\
\stackrel{\mathbb{1}}{I}\end{array}$ & 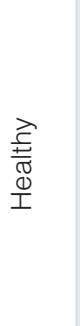 & 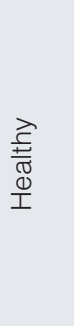 & $\begin{array}{l}\frac{0}{\partial} \\
\frac{0}{0} \\
\frac{1}{0} \\
0\end{array}$ & 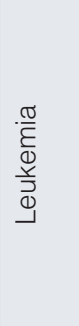 & $\begin{array}{l}\vec{I} \\
\frac{ \pm}{\widetilde{D}} \\
\stackrel{\oplus}{I}\end{array}$ \\
\hline 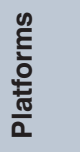 & 竞 & 竞 & 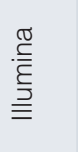 & 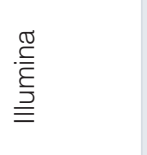 & $\begin{array}{l}\stackrel{\widetilde{c}}{\frac{.}{E}} \\
\stackrel{\equiv}{\equiv}\end{array}$ & 竞 & 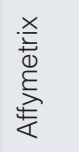 & 离 & 竞 & 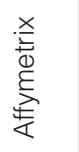 & 竞 & 竞 & 紊 & 站 & 竞 & 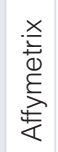 \\
\hline$E$ & 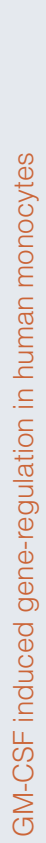 & 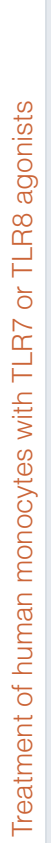 & 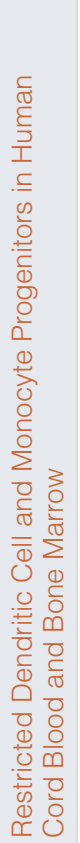 & 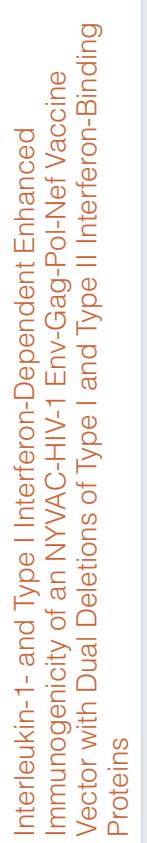 & 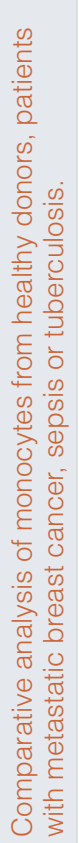 & 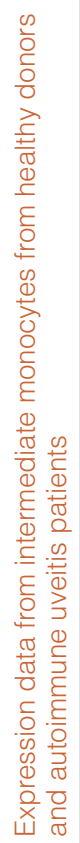 & 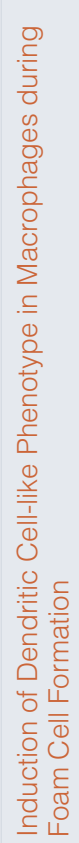 & 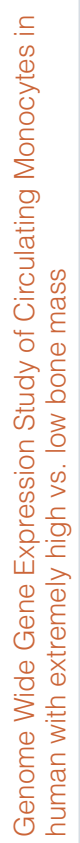 & 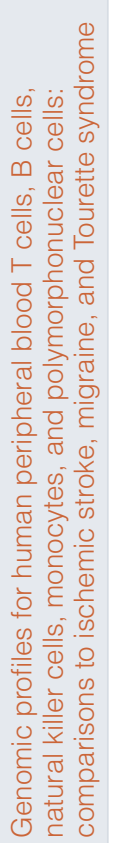 & 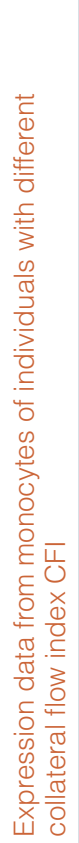 & 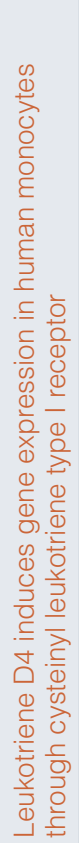 & 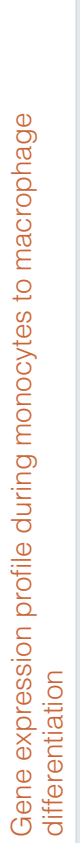 & 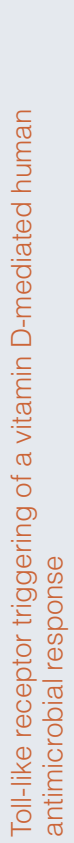 & 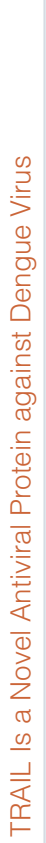 & 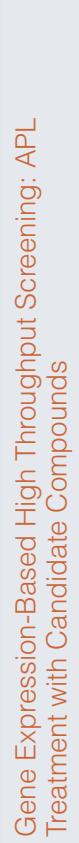 & 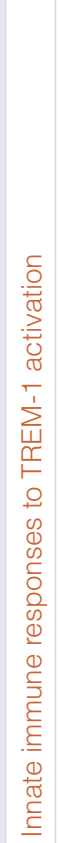 \\
\hline
\end{tabular}


context-rich environment. Selecting a gene from the rank ordered list on the left of the data-viewing interface will display its expression values graphically in the screen's central panel. Directly above the graphical display drop down menus give users the ability: a) To change how the gene list is ranked; this allows the user to change the method used to rank the genes, or to include only genes that are selected for specific biological interest; b) To change sample grouping (Group Set button), in some datasets a user can switch between groups based on cell type to groups based on disease type, for example; c) To sort individual samples within a group based on associated categorical or continuous variables (e.g. gender or age); d) To toggle between the bar chart view and a box plot view, with expression values represented as a single point for each sample. Samples are split into the same groups whether displayed as a bar chart or box plot; e) To provide a color legend for the sample groups; f) To select categorical information that is to be overlaid at the bottom of the graph. For example, the user can display gender or smoking status in this manner; g) To provide a color legend for the categorical information overlaid at the bottom of the graph; and h) To download the graph as a png image or csv file for performing a separate analysis. Measurements have no intrinsic utility in absence of contextual information. It is this contextual information that makes the results of a study or experiment interpretable. It is therefore important to capture, integrate and display information that will give users the ability to interpret data and gain new insights from it. We have organized this information under different tabs directly above the graphical display. The tabs can be hidden to make more room for displaying the data plots, or revealed by clicking on the blue "show info panel" button on the top right corner of the display. Information about the gene selected from the list on the left side of the display is available under the "Gene" tab. Information about the study is available under the "Study" tab. Information available about individual samples is provided under the "Sample" tab. Rolling the mouse cursor over a bar chart's element while displaying the "Sample" tab lists any clinical, demographic, or laboratory information available for the selected sample. Finally, the "Downloads" tab allows advanced users to retrieve the original dataset for analysis outside this tool. It also provides all available sample annotation data for use alongside the expression data in third party analysis software. Other functionalities are provided under the "Tools" dropdown menu located in the top right corner of the user interface. Some of the notable functionalities available through this menu include: a) Annotations, which provides access to all the ancillary information about the study, samples and dataset organized across different tabs; b) Cross-project view, which provides the ability for a given gene to browse through all available studies; c) Copy link, which generates a mini-URL encapsulating information about the display settings in use and that can be saved and shared with others (clicking on the envelope icon on the toolbar inserts the url in an email message via the local email client); and d) Chart options, which gives user the option to customize chart labels.

\section{Dataset validation}

Quality control checks were performed with the examination of profiles of relevant biological indicators. Known leukocyte markers were used, such as CD14, which is expressed by monocytes and macrophages; as well as markers that would indicate significant contamination of the sample by other leukocyte populations: such as CD3, a T-cells marker; CD19, a B-cell marker; CD56, an NK cell marker (Figure 3; The expression of the CD14 marker across all studies can be checked using the cross project functionality of GXB: http://monocyte.gxbsidra.org/dm3/geneBrowser/crossProject?prob eID=201743_at\&geneSymbol=CD14\&geneID=929). In addition, expression of the XIST transcripts, in which expression is genderspecific, was also examined to determine its concordance with demographic information provided with the GEO submission.

\section{SIDRA GXB Search: Sample Sels $\quad$ Reset}
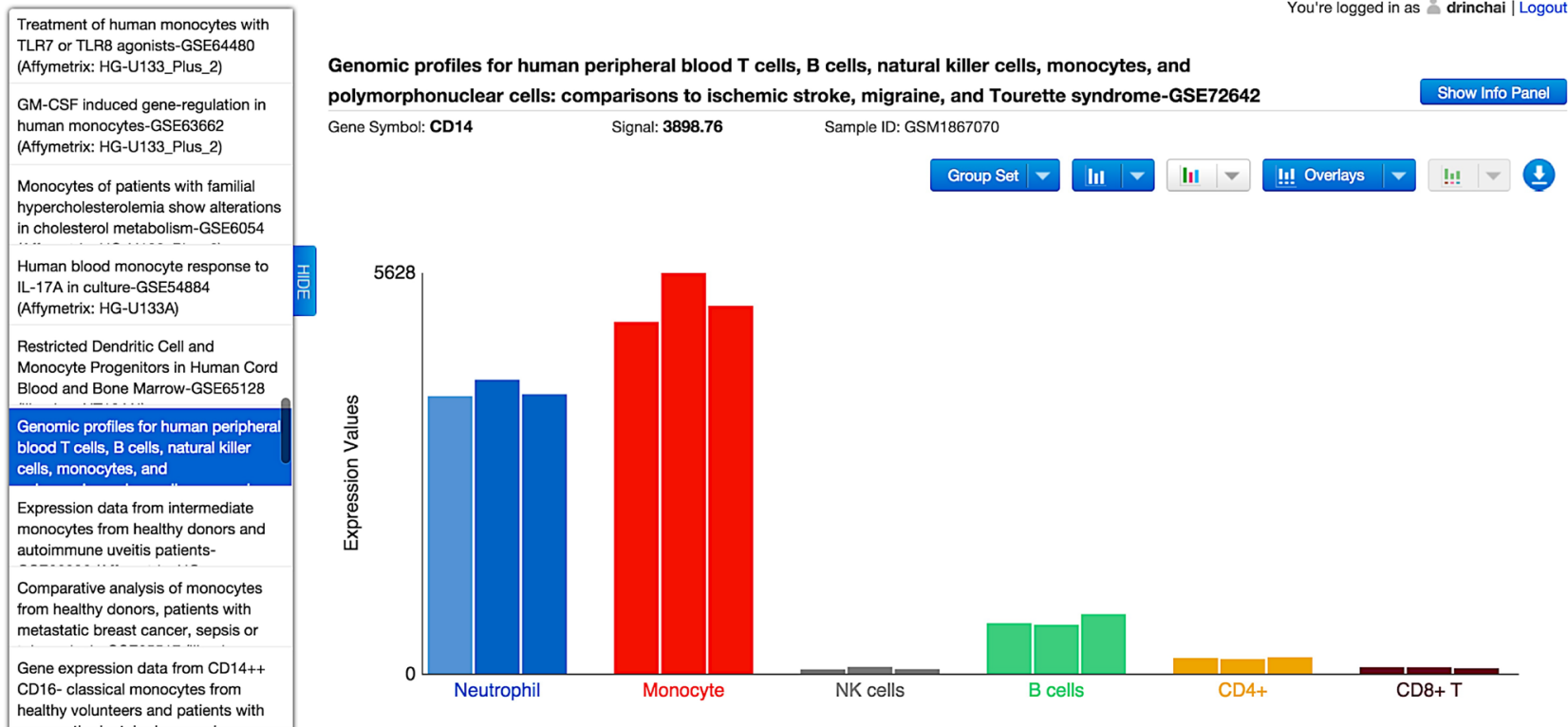

Figure 3. Illustrative example showing the abundance levels of CD14 transcripts across samples in a given study. The expression of this gene is indicative of the purity of primary human monocyte preparation; this marker is expected to be high in monocyte preparations and low in other leukocyte populations. In this view of the GXB expression of CD14 can be visualized across projects listed on the left. 


\section{Data availability}

All datasets included in our curated collection are also available publically via the NCBI GEO website: http://www.ncbi.nlm.nih. gov/geo/; and are referenced throughout the manuscript by their GEO accession numbers (e.g. GSE25913). Signal files and sample description files can also be downloaded from the GXB tool under the "downloads" tab.

\section{Author contributions}

DR: curated, uploaded and annotated datasets, and drafted the manuscript. SB: installed the software, uploaded datasets, programmed portions of the web application, and tested the software, and assisted in drafting the manuscript. SP: participated in the design of the software, programmed portions of the original web application, installed the software, and tested the software, and assisted in drafting the manuscript. CQ: participated in designed and programmed portions of the original web application, tested the software, and assisted in drafting the manuscript. DC: participated in software design, tested the software, and drafted the manuscript.

\section{Competing interests}

No competing interests were disclosed.

\section{Grant information}

DR, SB and DC received support from the Qatar Foundation.

I confirm that the funders had no role in study design, data collection and analysis, decision to publish, or preparation of the manuscript.

\section{Acknowledgement}

The authors would like to acknowledge all the investigators who decided to make their datasets publically available by sharing them in GEO.
1. Bennett L, Palucka AK, Arce $\mathrm{E}$, et al:: Interferon and granulopoiesis signatures in systemic lupus erythematosus blood. J Exp Med. 2003; 197(6): 711-23. PubMed Abstract | Publisher Full Text | Free Full Text

2. Griffiths MJ, Shafi MJ, Popper SJ, et al:: Genomewide analysis of the host response to malaria in Kenyan children. $J$ Infect Dis. 2005; 191(10): 1599-611. PubMed Abstract | Publisher Full Text

3. Mejias A, Dimo B, Suarez NM, et al:: Whole blood gene expression profiles to assess pathogenesis and disease severity in infants with respiratory syncytial virus infection. PLoS Med. 2013; 10(11): e1001549. PubMed Abstract | Publisher Full Text | Free Full Text

4. Moal V, Textoris J, Ben Amara A, et al:: Chronic hepatitis E virus infection is specifically associated with an interferon-related transcriptional program. $J$ Infect Dis. 2013; 207(1): 125-32. PubMed Abstract | Publisher Full Text

5. Mostafavi S, Battle A, Zhu X, et al:: Type I interferon signaling genes in recurrent major depression: increased expression detected by whole-blood RNA sequencing. Mol Psychiatry. 2014; 19(12): 1267-74 PubMed Abstract | Publisher Full Text

6. Novershtern N, Subramanian A, Lawton LN, et al.: Densely interconnected transcriptional circuits control cell states in human hematopoiesis. Cell. 2011; 144(2): 296-309.

PubMed Abstract | Publisher Full Text | Free Full Text

7. Panelli MC, Wang $E$, Phan $G$, et al: Gene-expression profiling of the response of peripheral blood mononuclear cells and melanoma metastases to systemic IL-2 administration. Genome Biol. 2002; 3(7): RESEARCH0035. PubMed Abstract | Publisher Full Text | Free Full Text

8. Pascual V, Allantaz F, Patel P, et al.: How the study of children with rheumatic diseases identified interferon-alpha and interleukin-1 as novel therapeutic targets. Immunol Rev. 2008; 223(1): 39-59.

PubMed Abstract | Publisher Full Text | Free Full Text

9. Smih F, Desmoulin F, Berry M, et al.: Blood signature of pre-heart failure: a microarrays study. PLoS One. 2011; 6(6): e20414. PubMed Abstract | Publisher Full Text | Free Full Text

10. Stamova B, $\mathrm{Xu} \mathrm{H}$, Jickling G, et al:: Gene expression profiling of blood for the prediction of ischemic stroke. Stroke. 2010; 41(10): 2171-7. PubMed Abstract | Publisher Full Text | Free Full Text

11. Berry MP, Graham CM, McNab FW, et al:: An interferon-inducible neutrophildriven blood transcriptional signature in human tuberculosis. Nature. 2010; 466(7309): 973-7.

PubMed Abstract | Publisher Full Text | Free Full Text

12. Martínez-Llordella M, Lozano JJ, Puig-Pey I, et al:: Using transcriptional profiling to develop a diagnostic test of operational tolerance in liver transplant recipients. J Clin Invest. 2008; 118(8): 2845-57. PubMed Abstract | Publisher Full Text | Free Full Text

13. Newell KA, Asare A, Kirk AD, et al:: Identification of a B cell signature associated with renal transplant tolerance in humans. J Clin Invest. 2010; 120(6): 1836-47.

PubMed Abstract | Publisher Full Text | Free Full Text
14. Gaucher D, Therrien R, Kettaf N, et al.: Yellow fever vaccine induces integrated multilineage and polyfunctional immune responses. J Exp Med. 2008; 205(13) 3119-31.

PubMed Abstract | Publisher Full Text | Free Full Text

15. Hecker M, Hartmann C, Kandulski O, et al.: Interferon-beta therapy in multiple sclerosis: the short-term and long-term effects on the patients' individual gene expression in peripheral blood. Mol Neurobiol. 2013; 48(3): 737-56. PubMed Abstract | Publisher Full Text

16. Li S, Rouphael N, Duraisingham S, et al:: Molecular signatures of antibody responses derived from a systems biology study of five human vaccines. Nat Immunol. 2014; 15(2): 195-204. PubMed Abstract | Publisher Full Text | Free Full Text

17. Obermoser G, Presnell S, Domico K, et al.: Systems scale interactive exploration reveals quantitative and qualitative differences in response to influenza and pneumococcal vaccines. Immunity. 2013; 38(4): 831-44. PubMed Abstract | Publisher Full Text | Free Full Text

18. Oswald M, Curran ME, Lamberth SL, et al:: Modular analysis of peripheral blood gene expression in rheumatoid arthritis captures reproducible gene expression changes in tumor necrosis factor responders. Arthritis Rheumatol. 2015; 67(2): 344-51.

PubMed Abstract | Publisher Full Text | Free Full Text

19. Querec TD, Akondy RS, Lee EK, et al.: Systems biology approach predicts immunogenicity of the yellow fever vaccine in humans. Nat Immunol. 2009; 10(1): 116-25.

PubMed Abstract | Publisher Full Text | Free Full Text

20. Speake C, Presnell S, Domico K, et al:: An interactive web application for the dissemination of human systems immunology data. J Transl Med. 2015; 13: 196 PubMed Abstract | Publisher Full Text | Free Full Text

21. Auffray C, Sieweke MH, Geissmann F: Blood monocytes: development, heterogeneity, and relationship with dendritic cells. Annu Rev Immunol. 2009; 27: 669-92.

PubMed Abstract | Publisher Full Text

22. van Furth R, Cohn ZA, Hirsch JG, et al.: The mononuclear phagocyte system: a new classification of macrophages, monocytes, and their precursor cells. Bull World Health Organ. 1972; 46(6): 845-52. PubMed Abstract | Free Full Text

23. Narni-Mancinelli E, Soudja SM, Crozat K, et al.: Inflammatory monocytes and neutrophils are licensed to kill during memory responses in vivo. PLOS Pathog. 2011; 7(12): e1002457

PubMed Abstract | Publisher Full Text | Free Full Text

24. Grimm MJ, Vethanayagam RR, Almyroudis NG, et al: Monocyte- and macrophagetargeted NADPH oxidase mediates antifungal host defense and regulation of acute inflammation in mice. $J$ Immunol. 2013; 190(8): 4175-84. PubMed Abstract | Publisher Full Text | Free Full Text

25. Fang FC: Antimicrobial reactive oxygen and nitrogen species: concepts and controversies. Nat Rev Microbiol. 2004; 2(10): 820-32. PubMed Abstract | Publisher Full Text

26. Dinauer MC, Deck MB, Unanue ER: Mice lacking reduced nicotinamide adenine 
dinucleotide phosphate oxidase activity show increased susceptibility to early infection with Listeria monocytogenes. J Immunol. 1997; 158(12): 5581-3. PubMed Abstract

27. Endres R, Luz A, Schulze $H$, et al.: Listeriosis in $p 47^{\text {phox }-/}$ and $T R p 55^{-/}$mice: protection despite absence of ROI and susceptibility despite presence of RNI. Immunity. 1997; 7(3): 419-32.

PubMed Abstract | Publisher Full Text

28. Aratani $\mathrm{Y}$, Koyama $\mathrm{H}$, Nyui $\mathrm{S}$, et al.: Severe impairment in early host defense against Candida albicans in mice deficient in myeloperoxidase. Infect Immun. 1999; 67(4): 1828-36.

PubMed Abstract | Free Full Text

29. Albrecht $D$, Jungi TW: Luminol-enhanced chemiluminescence induced in peripheral blood-derived human phagocytes: obligatory requirement of myeloperoxidase exocytosis by monocytes. J Leukoc Biol. 1993; 54(4): 300-6. PubMed Abstract

30. Gavrilin MA, Bouakl IJ, Knatz NL, et al.: Internalization and phagosome escape required for Francisella to induce human monocyte IL-1beta processing and release. Proc Natl Acad Sci U S A. 2006; 103(1): 141-6. PubMed Abstract | Publisher Full Text | Free Full Text

31. Serbina NV, Jia T, Hohl TM, et al:: Monocyte-mediated defense against microbial pathogens. Annu Rev Immunol. 2008; 26: 421-52. pabogens. Annu Rev Immunol. 2008; 26: 421-52.

32. Ancuta $\mathrm{P}$, Liu KY, Misra V, et al.: Transcriptional profiling reveals developmental relationship and distinct biological functions of CD16+ and CD16- monocyte subsets. BMC Genomics. 2009; 10: 403. PubMed Abstract | Publisher Full Text | Free Full Text

33. Frankenberger M, Hofer TP, Marei A, et al.: Transcript profiling of CD16-positive monocytes reveals a unique molecular fingerprint. Eur J Immunol. 2012; 42(4): 957-74.

PubMed Abstract | Publisher Full Text

34. Wong KL, Tai JJ, Wong WC, et al:: Gene expression profiling reveals the defining features of the classical, intermediate, and nonclassical human monocyte subsets. Blood. 2011; 118(5): e16-31. PubMed Abstract | Publisher Full Text

35. Zhu Y, Davis S, Stephens R, et al:: GEOmetadb: powerful alternative search engine for the Gene Expression Omnibus. Bioinformatics. 2008; 24(23): 2798-800. PubMed Abstract | Publisher Full Text | Free Full Text

36. Banchereau R, Baldwin N, Cepika AM, et al:: Transcriptional specialization of human dendritic cell subsets in response to microbial vaccines. Nat Commun. 2014; 5 : 5283

PubMed Abstract | Publisher Full Text | Free Full Text

37. Iwata M, Sandstrom RS, Delrow JJ, et al.: Functionally and phenotypically distinct subpopulations of marrow stromal cells are fibroblast in origin and induce different fates in peripheral blood monocytes. Stem Cells Dev. 2014; 23(7): 729-40.

PubMed Abstract | Publisher Full Text | Free Full Text

38. Llaverias G, Pou J, Ros E, et al.: Monocyte gene-expression profile in men with familial combined hyperlipidemia and its modification by atorvastatin treatment. Pharmacogenomics. 2008; 9(8): 1035-54. PubMed Abstract | Publisher Full Text

39. Maouche S, Poirier O, Godefroy T, et al:: Performance comparison of two microarray platforms to assess differential gene expression in human monocyte and macrophage cells. BMC Genomics. 2008; 9: 302 PubMed Abstract | Publisher Full Text | Free Full Text

40. Hu X, Chung AY, Wu I, et al:: Integrated regulation of Toll-like receptor responses by Notch and interferon-gamma pathways. Immunity. 2008; 29(5): 691-703. PubMed Abstract | Publisher Full Text | Free Full Text

41. Wrzesinski SH, Fisher JL, Ernstoff MS: Genetic profiles of plasmacytoid (BDCA-4 expressing) DC subtypes-clues to DC subtype function in vivo. Exp Hemato Oncol. 2013; 2(1): 8

PubMed Abstract | Publisher Full Text | Free Full Text

42. Butchar JP, Cremer TJ, Clay CD, et al:: Microarray analysis of human monocytes infected with Francisella tularensis identifies new targets of host response subversion. PLoS One. 2008; 3(8): e2924.

PubMed Abstract | Publisher Full Text | Free Full Text

43. Boomgaarden I, Egert S, Rimbach G, et al.: Quercetin supplementation and its effect on human monocyte gene expression profiles in vivo. Br J Nutr. 2010; 104(3): 336-45.

PubMed Abstract | Publisher Full Text

44. Karlsson KR, Cowley S, Martinez FO, et al:: Homogeneous monocytes and macrophages from human embryonic stem cells following coculture-free differentiation in M-CSF and IL-3. Exp Hematol. 2008; 36(9): 1167-75. PubMed Abstract | Publisher Full Text | Free Full Text

45. Szanto A, Balint BL, Nagy ZS, et al.: STAT6 transcription factor is a facilitator of the nuclear receptor PPAR $\gamma$-regulated gene expression in macrophages and dendritic cells. Immunity. 2010; 33(5): 699-712. PubMed Abstract | Publisher Full Text | Free Full Text

46. Poliska S, Csanky E, Szanto A, et al.: Chronic obstructive pulmonary disease-specific gene expression signatures of alveolar macrophages as well as peripheral blood monocytes overlap and correlate with lung function. Respiration. 2011; 81(6): 499-510. PubMed Abstract | Publisher Full Text
47. Strunnikova NV, Barb J, Sergeev YV, et al.: Loss-of-function mutations in Rab escort protein 1 (REP-1) affect intracellular transport in fibroblasts and monocytes of choroideremia patients. PLoS One. 2009; 4(12): e8402. PubMed Abstract | Publisher Full Text | Free Full Text

48. Ingersoll MA, Spanbroek R, Lottaz C, et al:: Comparison of gene expression profiles between human and mouse monocyte subsets. Blood. 2010; 115(3): e10-9.

PubMed Abstract | Publisher Full Text | Free Full Text

49. Fuentes-Duculan J, Suárez-Fariñas M, Zaba LC, et al:: A subpopulation of CD163-positive macrophages is classically activated in psoriasis. $J$ Invest Dermatol. 2010; 130(10): 2412-22.

PubMed Abstract | Publisher Full Text | Free Full Text

50. Semnani RT, Keiser PB, Coulibaly YI, et al.: Filaria-induced monocyte dysfunction and its reversal following treatment. Infect Immun. 2006; 74(8): 4409-17.

PubMed Abstract | Publisher Full Text | Free Full Text

51. Schmelzer C, Niklowitz P, Okun JG, et al:: Ubiquinol-induced gene expression signatures are translated into altered parameters of erythropoiesis and reduced low density lipoprotein cholesterol levels in humans. IUBMB Life. 2011; 63(1): 42-8

PubMed Abstract | Publisher Full Text

52. Smythies LE, Shen R, Bimczok D, et al.: Inflammation anergy in human intestinal macrophages is due to Smad-induced lkappaBalpha expression and NF-kappaB inactivation. J Biol Chem. 2010; 285(25): 19593-604. PubMed Abstract | Publisher Full Text | Free Full Text

53. Grigoryev YA, Kurian SM, Avnur Z, et al.: Deconvoluting post-transplant immunity: cell subset-specific mapping reveals pathways for activation and expansion of memory T, monocytes and B cells. PLoS One. 2010; 5(10): e13358.

PubMed Abstract | Publisher Full Text | Free Full Text

54. Chen LY, Eberlein M, Alsaaty $\mathrm{S}$, et al:: Cooperative and redundant signaling of leukotriene $\mathrm{B}_{4}$ and leukotriene $\mathrm{D}_{4}$ in human monocytes. Allergy. 2011; 66(10): 1304-11

PubMed Abstract | Publisher Full Text | Free Full Text

55. Liu F, Lei W, O'Rourke JP, et al:: Oncogenic mutations cause dramatic, qualitative changes in the transcriptional activity of c-Myb. Oncogene. 2006; 25(5): 795-805.

PubMed Abstract | Publisher Full Text

56. Allantaz $F$, Cheng DT, Bergauer $T$, et al.: Expression profiling of human immune cell subsets identifies miRNA-mRNA regulatory relationships correlated with cell type specific expression. PLOS One. 2012; 7(1): e29979. PubMed Abstract | Publisher Full Text | Free Full Text

57. Santer DM, Wiedeman AE, Teal TH, et al:: Plasmacytoid dendritic cells and C1q differentially regulate inflammatory gene induction by lupus immune complexes. J Immunol. 2012; 188(2): 902-15.

PubMed Abstract | Publisher Full Text | Free Full Text

58. Hulsmans M, Geeraert B, De Keyzer D, et al:: Interleukin-1 receptor-associated kinase- 3 is a key inhibitor of inflammation in obesity and metabolic syndrome. PLoS One. 2012; 7(1): e30414.

PubMed Abstract | Publisher Full Text | Free Full Text

59. Zhen A, Krutzik SR, Levin BR, et al:: CD4 ligation on human blood monocytes triggers macrophage differentiation and enhances HIV infection. J Virol. 2014; 88(17): 9934-46.

PubMed Abstract | Publisher Full Text | Free Full Text

60. Irvine KM, Gallego $\mathrm{P}, \mathrm{An} \mathrm{X}$, et al:: Peripheral blood monocyte gene expression profile clinically stratifies patients with recent-onset type 1 diabetes. Diabetes. 2012; 61(5): 1281-90.

PubMed Abstract | Publisher Full Text | Free Full Text

61. Schenk M, Krutzik SR, Sieling PA, et al:: NOD2 triggers an interleukin-32-dependent human dendritic cell program in leprosy. Nat Med. 2012; 18(4): 555-63. PubMed Abstract | Publisher Full Text | Free Full Text

62. Chauncey KM, Lopez MC, Sidhu G, et al.: Bacillus anthracis' lethal toxin induces broad transcriptional responses in human peripheral monocytes. $B M C$ Immunol. 2012; 13: 33.

PubMed Abstract | Publisher Full Text | Free Full Text

63. Henig N, Avidan N, Mandel I, et al.: Interferon-beta induces distinct gene expression response patterns in human monocytes versus T cells. PLoS One. 2013; 8(4): e62366.

PubMed Abstract | Publisher Full Text | Free Full Text

64. Haniffa M, Shin A, Bigley V, et al:: Human tissues contain CD141 ${ }^{\text {hi }}$ crosspresenting dendritic cells with functional homology to mouse $\mathrm{CD}_{103}$ nonlymphoid dendritic cells. Immunity. 2012; 37(1): 60-73. PubMed Abstract | Publisher Full Text | Free Full Text

65. Hou W, Gibbs JS, Lu X, et al.: Viral infection triggers rapid differentiation of human blood monocytes into dendritic cells. Blood. 2012; 119(13): 3128-31. PubMed Abstract | Publisher Full Text | Free Full Text

66. Ganesh K, Das A, Dickerson R, et al:: Prostaglandin $\mathrm{E}_{2}$ induces oncostatin $M$ expression in human chronic wound macrophages through Axl receptor tyrosine kinase pathway. J Immunol. 2012; 189(5): 2563-73. PubMed Abstract | Publisher Full Text | Free Full Text

67. Chittezhath M, Dhillon MK, Lim JY, et al:: Molecular profiling reveals a tumorpromoting phenotype of monocytes and macrophages in human cancer 
progression. Immunity. 2014; 41(5): 815-29. PubMed Abstract | Publisher Full Text

68. Ismail N, Wang $\mathrm{Y}$, Dakhlallah $\mathrm{D}$, et al:: Macrophage microvesicles induce macrophage differentiation and miR-223 transfer. Blood. 2013; 121(6): 984-95. PubMed Abstract | Publisher Full Text | Free Full Text

69. Hyder LA, Gonzalez J, Harden JL, et al:: TREM-1 as a potential therapeutic target in psoriasis. J lnvest Dermatol. 2013: 133(7): 1742-51. PubMed Abstract | Publisher Full Text | Free Full Text

70. Teles RM, Graeber TG, Krutzik SR, et al:: Type I interferon suppresses type II interferon-triggered human anti-mycobacterial responses. Science. 2013; 339(6126): 1448-53.

PubMed Abstract | Publisher Full Text | Free Full Text

71. Wheelwright M, Kim EW, Inkeles MS, et al:: All-trans retinoic acid-triggered antimicrobial activity against Mycobacterium tuberculosis is dependent on NPC2. J Immunol. 2014; 192(5): 2280-90.

PubMed Abstract | Publisher Full Text | Free Full Text

72. Shalova IN, Lim JY, Chittezhath M, et al.: Human monocytes undergo functiona re-programming during sepsis mediated by hypoxia-inducible factor- $1 \alpha$. Immunity. 2015; 42(3): 484-98.

PubMed Abstract | Publisher Full Text

73. Hamm A, Prenen H, Van Delm W, et al.: Tumour-educated circulating monocytes are powerful candidate biomarkers for diagnosis and disease follow-up of colorectal cancer. Gut. 2015; pii: gutjnl-2014-308988. PubMed Abstract | Publisher Full Text

74. Martinez FO, Helming L, Milde R, et al.: Genetic programs expressed in resting and IL-4 alternatively activated mouse and human macrophages: similarities and differences. Blood. 2013; 121(9): e57-69.

PubMed Abstract | Publisher Full Text

75. Levine AJ, Horvath S, Miller EN, et al:: Transcriptome analysis of HIV-infected peripheral blood monocytes: gene transcripts and networks associated with neurocognitive functioning. J Neuroimmunol. 2013; 265(1-2): 96-105. PubMed Abstract | Publisher Full Text | Free Full Text

76. Radom-Aizik S, Zaldivar FP Jr, Haddad F, et al:: Impact of brief exercise on circulating monocyte gene and microRNA expression: implications for atherosclerotic vascular disease. Brain Behav Immun. 2014; 39: 121-9. PubMed Abstract | Publisher Full Text | Free Full Text

77. Kyogoku C, Smiljanovic B, Grün JR, et al:: Cell-specific type I IFN signatures in autoimmunity and viral infection: what makes the difference? PLOS One. 2013; 8(12): e83776.

PubMed Abstract | Publisher Full Text | Free Full Text

78. Tilton JC, Johnson AJ, Luskin MR, et al: Diminished production of monocyte proinflammatory cytokines during human immunodeficiency virus viremia is mediated by type I interferons. J Virol. 2006; 80(23): 11486-97. PubMed Abstract | Publisher Full Text | Free Full Text

79. Wu JQ, Sassé TR, Saksena MM, et al: Transcriptome analysis of primary monocytes from HIV-positive patients with differential responses to antiretroviral therapy. Virol J. 2013; 10: 361.

PubMed Abstract | Publisher Full Text | Free Full Text

80. Sharma S, Jin Z, Rosenzweig E, et al:: Widely divergent transcriptional patterns between SLE patients of different ancestral backgrounds in sorted immune cell populations. J Autoimmun. 2015; 60: 51-8. PubMed Abstract | Publisher Full Text | Free Full Text

81. Laudanski K, Miller-Graziano C, Xiao W, et al:: Cell-specific expression and pathway analyses reveal alterations in trauma-related human $\mathrm{T}$ cell and monocyte pathways. Proc Natl Acad Sci U S A. 2006; 103(42): 15564-9. PubMed Abstract | Publisher Full Text | Free Full Text
82. Reynolds LM, Taylor JR, Ding J, et al:: Age-related variations in the methylome associated with gene expression in human monocytes and T cells. Nat Commun. 2014; 5: 5366.

PubMed Abstract | Publisher Full Text | Free Full Text

83. Mosig S, Rennert K, Büttner $\mathrm{P}$, et al:: Monocytes of patients with familial hypercholesterolemia show alterations in cholesterol metabolism. BMC Med Genomics. 2008; 1: 60

PubMed Abstract | Publisher Full Text | Free Full Text

84. Däbritz J, Weinhage T, Varga G, et al:: Reprogramming of monocytes by GM-CSF contributes to regulatory immune functions during intestinal inflammation. J Immunol. 2015; 194(5): 2424-38.

PubMed Abstract | Publisher Full Text

85. Elavazhagan S, Fatehchand K, Santhanam V, et al:: Granzyme B expression is enhanced in human monocytes by TLR8 agonists and contributes to antibodydependent cellular cytotoxicity. J Immunol. 2015; 194(6): 2786-95. PubMed Abstract | Publisher Full Text | Free Full Text

86. Lee J, Breton G, Oliveira TY, et al:: Restricted dendritic cell and monocyte progenitors in human cord blood and bone marrow. J Exp Med. 2015; 212(3): 385-99.

PubMed Abstract | Publisher Full Text | Free Full Text

87. Bergenfelz C, Larsson AM, von Stedingk K, et al.: Systemic Monocytic-MDSC Are Generated from Monocytes and Correlate with Disease Progression in Breast Cancer Patients. PLoS One. 2015; 10(5): e0127028. PubMed Abstract | Publisher Full Text | Free Full Text

88. Liu B, Dhanda A, Hirani S, et al:: CD14 ${ }^{++}$CD16 ${ }^{+}$Monocytes Are Enriched by Glucocorticoid Treatment and Are Functionally Attenuated in Driving Effector T Cell Responses. J Immunol. 2015; 194(11): 5150-60. PubMed Abstract | Publisher Full Text | Free Full Text

89. Cho HJ, Shashkin P, Gleissner CA, et al: Induction of dendritic cell-like phenotype in macrophages during foam cell formation. Physiol Genomics. 2007; 29(2): 149-60.

PubMed Abstract | Publisher Full Text

90. Du X, Tang $\mathrm{Y}, \mathrm{Xu} \mathrm{H}$, et al: Genomic profiles for human peripheral blood T cells, B cells, natural killer cells, monocytes, and polymorphonuclear cells: comparisons to ischemic stroke, migraine, and Tourette syndrome. Genomics. 2006; 87(6): 693-703.

PubMed Abstract | Publisher Full Text

91. Meier $\mathrm{P}$, Antonov J, Zbinden $\mathrm{R}$, et al.: Non-invasive gene-expression-based detection of well-developed collateral function in individuals with and without coronary artery disease. Heart. 2009; 95(11): 900-8. PubMed Abstract | Publisher Full Text

92. Woszczek $G$, Chen LY, Nagineni $S$, et al:: Leukotriene $D_{4}$ induces gene expression in human monocytes through cysteinyl leukotriene type I receptor. $J$ Allergy Clin Immunol. 2008; 121(1): 215-21.e1. PubMed Abstract | Publisher Full Text

93. Liu PT, Stenger S, Li H, et al:: Toll-like receptor triggering of a vitamin D-mediated human antimicrobial response. Science. 2006; 311(5768): 1770-3. PubMed Abstract | Publisher Full Text

94. Stegmaier K, Ross KN, Colavito SA, et al.: Gene expression-based highthroughput screening(GE-HTS) and application to leukemia differentiation. Nat Genet. 2004; 36(3): 257-63. PubMed Abstract | Publisher Full Text

95. Dower K, Ellis DK, Saraf K, et al:: Innate immune responses to TREM-1 activation: overlap, divergence, and positive and negative cross-talk with bacterial lipopolysaccharide. J Immunol. 2008; 180(5): 3520-34. PubMed Abstract | Publisher Full Text 


\title{
Open Peer Review
}

\section{Current Peer Review Status:}

\section{Version 1}

Reviewer Report 21 March 2016

https://doi.org/10.5256/f1000research.8800.r12769

(C) 2016 Chen $\mathbf{P}$ et al. This is an open access peer review report distributed under the terms of the Creative Commons Attribution License, which permits unrestricted use, distribution, and reproduction in any medium, provided the original work is properly cited.

\author{
Ping Chen \\ National Eye Institute (NEI), National Institutes of Health, Besthesda, MD, USA \\ David Kuo \\ ${ }^{1}$ National Eye Institute (NEI), National Institutes of Health, Bethesda, MD, USA \\ 2 University of California San Diego, La Jolla, CA, USA
}

\section{General Comments}

Modern genomics, especially with the emergence of high-throughput next-generation sequencing, is generating data at such a rapid rate that new tools for organizing, visualizing, sharing, and integrating heterogeneous data in the context of scientific information are needed for scientists to efficiently use these published data. The Chaussabel group has recently developed an interactive data browsing and visualization web application, the Gene Expression Browser (GXB), to address this problem.

In this data note, Dr. Rinchai et al. report a compendium of ninety-six curated human monocyte transcriptome datasets from GEO spanning a broad range of diseases, cell types, and experiments. These datasets were then uploaded to the Gene Expression Browser for exploratory data analysis and dataset validation. The Gene Expression Browser should prove very useful for investigating large datasets; however, I have several questions and comments regarding the curated data itself:

Title:

The novel aspect and apparent emphasis of this data note is using the Gene Expression Browser to more easily explore the curated ninety-six datasets. But the current title emphasizes the key information on fostering the knowledge discovery. Please consider rephrasing it by focusing on the monocyte datasets and web application.

\section{Introduction:}

As the Gene Expression Browser has been described in detail previously, the emphasis of this data note should be on the curated data. It would be helpful to discuss the motivation for creating this 
particular compendium of monocyte transcriptome datasets as well as the intended use of the curated data given the breadth and heterogeneity of diseases, cell types, and experiments that it includes.

\section{Methods:}

1. Please elaborate more specifically on how the datasets were curated. What were the eligibility criteria for inclusion into the compendium?

2. The table summarizing the published data can difficult to read due to its landscape orientation. Consider rotating the table from a landscape orientation to a portrait orientation.

3. In the right pie chart of Figure 2, there are twelve datasets studying primary monocytes; however, datasets classified as in vitro stimulation, infection, and monocyte subsets may also contain primary monocytes. Better categorization is needed.

4. Data validation is critical for verifying that a dataset is acceptable for use. The authors mention performing dataset validation but do not report the related results or summary of their validation. On page 9 , the process of assessing contamination by other leukocyte populations using surface markers should be done carefully as CD14 ${ }^{+}$monocytes do share surface marker CD4.

5. In Fig. 3, it is unclear whether the orange bar plot is referring to $\mathrm{CD} 4^{+} \mathrm{T}$ cells or $\mathrm{CD} 4^{+}$cells in general. They are different cell types.

Competing Interests: No competing interests were disclosed.

We confirm that we have read this submission and believe that we have an appropriate level of expertise to confirm that it is of an acceptable scientific standard, however we have significant reservations, as outlined above.

Author Response 29 Mar 2016

Darawan Rinchai, Sidra Medical and Research Center, Doha, Qatar

We thank the reviewers for their valuable feedback and suggestions to improve our manuscript.

Title:

Following the suggestion of the reviewers we changed the title of the manuscript to "A curated compendium of of transcriptome datasets of relevance to human monocyte immunobiology research".

Introduction:

Thanks for raising this point. We added a long paragraph and new references in the introduction to emphasize the role of monocyte across different diseases and the motivation for creating this compendium of monocyte transcriptome datasets.

Methods:

1. We have added information about how datasest were selected for inclusion in the collections in 
the methods section under the title "Identification of monocyte datasets"..."Using the search query, the results also returned a number of datasets that did not include profiles of monocytes but instead of "monocyte-derived dendritic cells" or "monocyte-derived macrophages". During our manual screen these were excluded as were studies employing monocytic cell lines. Only studies including primary human monocyte profiles were retained."...

2. We agree with the reviewer that presenting the table using landscape orientation makes it difficult to read. We therefore changed table format from landscape to portrait orientation.

3. Thank you for pointing this out. We changed the label on this figure to read "ex-vivo, no treatment". These include studies where monocytes were isolated from healthy subjects for comparison with other cell types, or evaluation of variation among healthy individuals.

4. Assessing contamination can indeed be difficult, especially using this type of data where celllevel information is lacking. We plan to explore with our bioinformatics collaborators the development of a "scoring" approach to better quantify potential contamination but this is not a simple matter to address. At this point we have simply verified for each dataset that expression of markers was consistent with grouping labels provided by depositors. We have added language in the manuscript to clarify this point.

5. Thank you for pointing out this typo on this label. This dataset focuses on genomic profile of human blood both CD4+ and CD8+T cells, B cells, NK cells monocytes and neutrophil. Figure 3 was corrected accordingly as shown in the new Figure 3.

Competing Interests: No competing interests were disclosed.

Reviewer Report 16 March 2016

\section{https://doi.org/10.5256/f1000research.8800.r12768}

(C) 2016 Pellegrini M. This is an open access peer review report distributed under the terms of the Creative Commons Attribution License, which permits unrestricted use, distribution, and reproduction in any medium, provided the original work is properly cited.

\section{Marc Pellegrini}

Division of Infection and Immunity, Walter and Eliza Hall Institute of Medical Research, Parkville, VIC, Australia

In this short descriptive report the authors put their published Gene Expression Browser tool to work in arranging several thousand transcriptome profiles obtained from public datasets that looked at monocyte immunology. They were able to compare groups of monocytes based on phenotypic attributes and rank gene expression. The authors provide a nice summary of the technique and validation. 
Competing Interests: No competing interests were disclosed.

I confirm that I have read this submission and believe that I have an appropriate level of expertise to confirm that it is of an acceptable scientific standard.

The benefits of publishing with F1000Research:

- Your article is published within days, with no editorial bias

- You can publish traditional articles, null/negative results, case reports, data notes and more

- The peer review process is transparent and collaborative

- Your article is indexed in PubMed after passing peer review

- Dedicated customer support at every stage

For pre-submission enquiries, contact research@f1000.com 